\title{
Control input separation by actuation mode expansion for flow control problems
}

\author{
Coşku Kasnakoğlu ${ }^{a *}$, Andrea Serrani ${ }^{\mathrm{b}}$ and Mehmet Önder Efe ${ }^{\mathrm{a}}$ \\ ${ }^{a}$ Department of Electrical \& Electronics Engineering, TOBB Economics and Technology University, \\ Ankara, Turkey; ${ }^{b}$ Department of Electrical and Computer Engineering, The Ohio State University, \\ Columbus, Ohio, USA
}

(Received 1 July 2007; final version received 15 December 2007)

\begin{abstract}
A control input separation method is proposed for reduced-order modelling in boundary control problems. The dynamics of flow systems are typically described by partial differential equations where the input affects the system through boundary conditions. From a control design perspective it is most desirable and natural to employ finite-dimensional representations in which the input enters the dynamics directly. The method proposed here to resolve the input from the boundary conditions is based on obtaining a proper orthogonal decomposition of the unforced flow of the system, and then augmenting this decomposition by optimally computed actuation modes, built using snapshots of the actuated flow. A reduced-order Galerkin model is then derived for this expansion, in which the input appears as an explicit term in the system dynamics. The model reduces exactly to the original baseline case under zero input conditions. The proposed method is then compared to an existing input separation technique, namely the sub-domain separation method. A boundary control example regarding the $2 \mathrm{D}$ incompressible Navier-Stokes equation is considered to illustrate the proposed method, where a controller is designed to achieve tracking of a desired 2D spatial profile for the flow velocity.
\end{abstract}

Keywords: control input separation; flow control; boundary control; proper orthogonal decomposition; Galerkin projection

\section{Introduction}

It is a characteristic of flow control problems that the control input is applied at the physical boundaries of the system. When the systems are modelled as partial differential equations, the control input is usually specified as a boundary condition, hence the terminology boundary control. Among the myriad of examples of boundary control, Smaoui (2005) analysed the dynamics of the forced Burgers' equation subject to both Neumann boundary conditions and periodic boundary conditions using boundary and distributed control. Hinze and Kunisch (2004) devised secondorder methods for open loop optimal boundary control problems governed by the non-stationary Navier-Stokes system. Non-linear boundary control of coupled Burgers' equations was studied by Kobayashi and Oya (2003). Park and Lee (2000) studied boundary control of the Navier-Stokes equation by empirical reduction of modes. Baramov et al. (2004) considered $H_{\infty}$ control of Navier-Stokes equations governing non-periodic 2D channel flow, significantly reducing unwanted disturbances. Global stabilisation of Burgers' equation by boundary control was studied by Krstic (1999), where non-linear boundary control laws were derived to achieve global asymptotic stability of viscous and inviscid Burgers' equation, using both Neumann and Dirichlet boundary control. In Aamo et al. (2003), enhancement of mixing was accomplished by implementing boundary control for Navier-Stokes equations describing 2D channel flow.

Typical techniques for model development in flow control problems are either directly based on flow physics or rely on system identification techniques from experimental data. Examples of the latter include Mongeau et al. (1998) and Kook et al. (2002), where open-loop transfer functions for low-speed flows at several different flow velocities are determined by observing the response to forcing. In Rowley and Williams (2003), a similar approach was followed, with the difference that frequency-response experiments were performed on the system stabilised with a known controller tuned to suppress cavity oscillations. A frequency-response identification using a leastsquares method was performed by Cattafesta et al. (1997) to identify the parameters of a discrete-time transfer function, and similar techniques were used by

\footnotetext{
*Corresponding author. Email: kasnakoglu@etu.edu.tr
} 
Cabell et al. (2002) to obtain very high-order models. Cattafesta et al. (1999) proposed an adaptive algorithm which could be used either for off-line identification, or to tune the parameters of a feedback controller. Discrete-time adaptive algorithms have been considered in Pillarisetti and Cattafesta (2001) and Kegerise et al. (2002). As to the modelling approaches using governing equations, Rowley et al. (2000, 2001), Lehmann et al. (2005), Smith et al. (2002), Glauser et al. (2004a, b) and Rempfer (2000) can be listed as typical examples sharing a common core methodology. An optimal set of basis vectors are built using proper orthogonal decomposition (POD) methods from snapshots of the flow collected from experimental data or direct numerical simulations. Then, the governing equations are projected onto the subspace spanned by the POD basis, a procedure known as Galerkin Projection (GP), which yields a finite-dimensional system that approximates the original system dynamics in an energy-optimal sense. The model obtained in this fashion is called a Galerkin model (GM), while the general methodology used to obtain finite-dimensional approximations of the original governing equations is termed model reduction. A detailed description of the POD-GP method can be found in Holmes et al. (1996). Reduced-order models that are derived using balanced truncation techniques are also of interest (Lawrence et al. 2005). In addition to the work listed above, standard references in the fields of boundary control, control of infinite-dimensional systems and model reduction techniques include Bensoussan et al. (1992), Curtain and Zwart (1995) and Burns and King (1998), Aamo and Krstic (2003), Antoulas (2005) and King et al. (2005).

When obtaining a reduced-order model, the control input must be separated from the boundary conditions, and its presence made explicit in the model. The method that takes boundary control to this more natural form for control systems design is referred to as input separation. Previous work on input separation includes Efe and Ozbay (2003a, b, 2004) where POD/GP Models are obtained for Burgers' equations and heat equations in which the control input, specified as a free boundary condition, is made to appear as an additive term in the differential equation by means of sub-domain separation. In Samimy et al. (2007), the same methodology has been applied to obtain experimentally-based reduced-order models of cavity flows, and employed for controller design. In the work by Camphouse (2005), reduced-order models are obtained with input separation performed through weak formulation of POD-based models.

Although the studies cited above provide a means of obtaining the input as an explicit term, there are still associated issues, and room for improvement. Usually the separation is performed a posteriori with respect to the generation of a POD basis. This results in the situation where the model is not reduced to the unforced baseline case when the input is set to zero. An additional problem inherent in the current methods is that POD bases do not capture the effect of the external forcing, but rather the dynamics of the input under which they were built. This limits the ability of the model to represent forcing conditions that are significantly different from those used for identification. A final issue is associated with underestimation of the control vector field in the reduced-order model, which creates a mismatch between values obtained in simulation for the control signal and its actual value to be used in experiments.

In this paper, a control input separation method is proposed that aims at addressing some of the issues mentioned above. The proposed method relies on an expansion of the flow field in terms of baseline POD modes and actuation modes, where the latter is obtained from actuated snapshots a posteriori to the baseline POD modes. Like the standard POD, the methodology yields an optimal selection of a finitedimensional approximation, in the sense that the energy of the actuated flow neglected by the expansion is minimised. The outcome is a finitedimensional system in which the input appears explicitly, and which reduces exactly to the dynamics obtained from the original POD expansion when the input vanishes.

The paper is organised as follows: Sections 2 and 3 provide an introduction and a brief review of classical POD/GP methods, respectively; in Section 4 the derivation of the actuation mode expansion is presented, and applied in Section 5 to reduced-order modelling; Section 6 compares the proposed method to an available technique for input separation, namely the sub-domain method; Section 7 illustrates the usefulness of the proposed approach in feedback control design by means an example regarding control of 2D incompressible Navier-Stokes equations; Section 8 provides concluding remarks.

\section{Problem description}

Consider a planar spatial domain $\Omega \subset \mathbb{R}^{2}$, and let $\mathbb{R}$ be a real Hilbert space with inner product $\langle\cdot, \cdot\rangle$ : $\mathbb{H} \times \mathbb{U} \rightarrow \mathbb{R}$ and induced norm $\|\cdot\|$. Throughout the paper, we will focus our attention to $\mathbb{H}=\mathcal{L}_{2}\left(\Omega, \mathbb{R}^{2}\right)$ with the standard inner product $\left\langle h_{1}, h_{2}\right\rangle=\int_{\Omega} h_{1}(x)^{\mathrm{T}}$ $h_{2}(x) \mathrm{d} x$ for $h_{1}, h_{2} \in \mathcal{L}_{2}$, although the results apply to other Hilbert spaces as well. Let $u(x, t)$ be a flow velocity field over $\Omega$, where $u: \Omega \times \mathbb{R}_{+} \rightarrow \mathbb{R}^{2}, u(\cdot, t) \in \mathbb{H}$, $u(x, \cdot) \in \mathcal{C}^{k}, k \geq 1$. Here, $t \in \mathbb{R}_{+}$is the temporal variable and $x \in \Omega$ is the spatial variable. The evolution of the 
flow field is governed by a partial differential equation (PDE) of the form

$$
\dot{u}=X(u),
$$

where the operator $X: \mathbb{W} \rightarrow \mathbb{U}$ includes spatial derivatives. Equation (1) is subject to the initial condition

$$
u(x, 0)=u_{\text {init }}(x),
$$

where $u_{\text {init }} \in \mathbb{H}$, and subject to the boundary conditions

$$
\left(B_{i}(u, \gamma)\right)(x, t)=b_{i}(x, t), \quad i=1 \ldots N_{b},
$$

where $x \in \partial \Omega, \quad t \in R_{+}, \quad B_{i}: \mathbb{H} \times \mathcal{C}^{k} \rightarrow \mathbb{H}, \quad b_{i} \in \mathbb{H}$, and $N_{b} \in N$. A control input $\gamma \in \mathbb{R}$ acts through the boundary conditions. The operator $B$ may include spatial derivatives.

The problem considered in this work is to develop an approach to obtaining reduced-order models of system (1)-(3) in a form that is useful for standard control system design, that is, a model having the property that the input $\gamma$ enters the dynamics directly. This means obtaining an ordinary differential equation of the form

$$
\dot{a}=f(a, \gamma), \quad a(0)=a_{0},
$$

where $f: \mathbb{R}^{N} \times \mathbb{R} \rightarrow \mathbb{R}^{N}$ and $a \in R^{N}$, in such a way that (4) approximates the original PDE (1)-(3) in some optimal sense. The proposed approach builds upon classical POD/GP techniques (Holmes et al. 1996), of which a brief review is provided in the following section.

\section{Review of classical POD/GP}

Denote by $u(x, t)$ the solution of the PDE (1)-(3). Let $u_{k}(x)=u\left(x, t_{k}\right)$ be a snapshot taken at time $t_{k}$ and let $\left\{u_{k}\right\}_{k=1}^{M} \subset \mathbb{W}$ be an ensemble of $M \in \mathbb{N}$ snapshots collected at times $\left\{t_{k}\right\}_{k=1}^{M}$. Let $u_{0}=E\left[\left\{u_{j}\right\}_{j=1}^{M}\right]$ where $E$ is the linear averaging operation $E\left[\left\{u_{j}\right\}_{j=1}^{M}\right]=$ $M^{-1} \Sigma_{j=1}^{M} w_{j} u_{j}$, for given weights $w_{j} \in \mathbb{R}_{+}$. As it is clear that the index for the snapshots ranges from 1 to $M$, we will write $E\left[u_{j}\right]$ instead of $E\left[\left\{u_{j}\right\}_{j=1}^{M}\right]$ for ease of notation. Among all subspaces $S \subset \mathbb{W}$ of a given dimension $N<M$, the one that minimises the averaged error

$$
J(S)=E\left[\left\|u_{j}-P_{S} u_{j}\right\|^{2}\right],
$$

where $P_{S}$ denotes projection onto $S$, is given by the subspace spanned by the orthonormal eigenfunctions $\phi_{i}$ corresponding to the $N$ largest non-zero eigenvalues of the linear operator $R: \mathbb{H} \rightarrow S$ given by the correlation tensor

$$
R=E\left[u_{j} \otimes u_{j}^{*}\right]
$$

Here $u_{j}^{*}$ is the dual vector of $u_{j}$ in $\mathbb{R}$, whereas the tensor product $\otimes$ is defined as $(v \otimes \alpha) w=v \alpha(w)$ for $v, w \in \mathbb{M}$ and $\alpha \in \mathbb{U}^{*}$; (see Holmes et al. (1996) and Rowley et al. (2004)). The vectors $\phi_{i}, i=1, \ldots, N$, are called the POD modes of the ensemble. Although the problem as posed is infinite-dimensional, it can be shown that it suffices to solve the finite $M$-dimensional eigenvalue problem

$$
C \phi_{i}=\lambda_{i} \phi_{i}
$$

where $\phi_{i}=\left(\phi_{i 1}, \ldots, \phi_{i M}\right)$, and $C \in \mathbb{R}^{M \times M}$ is the correlation matrix with entries $C_{i j}=\left\langle u_{j}, u_{i}\right\rangle$. This method is known as the method of snapshots (Sirovich 1987). Once the POD modes $\phi_{i}$ are obtained as described here, the flow can be represented as a finite dimensional approximation as

$$
u(x, t) \approx \sum_{i=1}^{N} a_{i}(t) \phi_{i}(x) .
$$

It is also customary to remove the mean value $u_{0}$ from the snapshots prior to the calculation on the POD modes. As a result, instead of $\left\{u_{k}\right\}_{k=1}^{M}$, one carries out the above procedure for $\left\{u_{k}-u_{0}\right\}_{k=1}^{M}$, obtaining the expansion

$$
u(x, t) \approx u_{0}(x)+\sum_{i=1}^{N} a_{i}(t) \phi_{i}(x) .
$$

Whenever convenient, we will adopt Einstein notation, and write the above expression as $u(x, t) \approx u_{0}(x)+a_{i}(t)$ $\phi_{i}(x)$, with the understanding that terms with repeated indices are summed over their possible values.

Let $S:=\operatorname{span}\left\{\phi_{1}, \ldots, \phi_{N}\right\}$ be the subspace spanned by the POD basis vectors, and define the linear variety $V:=u_{0}+S$. A dynamical system

$$
\dot{r}=X_{V}(r)
$$

on $V$ that approximates (1) can be obtained by Galerkin projection as

$$
X_{V}(r)=P_{V} X(r),
$$

where the projection operator from $\mathbb{H}$ to $V$ is defined as $P_{V} \quad w=u_{0}+P_{S}\left(w-u_{0}\right)$, for $w \in \mathbb{H}$. Since the $i$-th component of $P_{V} w$ is $\left\{P_{V} w\right\}_{i}=\left\langle w-u_{0}, \phi_{i}\right\rangle+u_{0_{i}}$, the components of the vector field of the Galerkin system are given by

$$
\left\{P_{V} X(r)\right\}_{i}=\left\langle X(r)-u_{0}, \phi_{i}\right\rangle+u_{0 i} .
$$

Figure 1 provides a graphical illustration of the projection operator $P_{V}$. The Galerkin projection is optimal in the sense that it minimises $\left\|X_{V}(r)-X(r)\right\|$. Since $X_{V}(r)-X(r) \perp S$, one can write

$$
\left\langle X_{V}(r)-X(r), \phi_{i}\right\rangle=0, \quad i=1 \ldots N .
$$




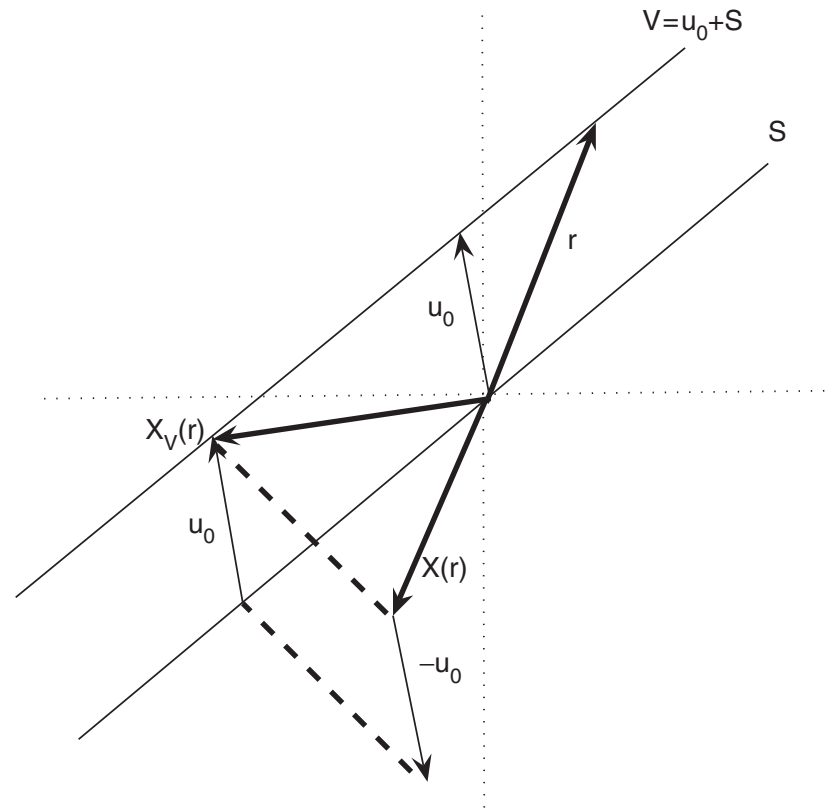

Figure 1. Illustration of Galerkin projection from $\mathbb{H}$ to $V$.

Substituting $r=u_{0}+a_{j} \phi_{j}$ into (6) above, and using (5) and orthogonality, one obtains the set of non-linear ODEs

$$
\dot{a}_{k}=\left\langle X(r), \phi_{k}\right\rangle, \quad k=1 \ldots N .
$$

At this stage, the effect of actuation is still buried in the boundary conditions (3), and does not appear explicitly in (7). In the next section we will investigate the method proposed to remedy this situation.

\section{Actuation mode}

Let $S=\operatorname{span}\left\{\phi_{i}\right\}_{i=1}^{N}$ be a set of $N$ POD modes obtained from unforced snapshots (that is, for $\gamma=0$ ) using the POD procedure described in $\S 3$. These modes will be called baseline POD modes. Let $\left\{\left(u_{k}, \gamma_{k}\right)\right\}_{k=1}^{M} \subset \mathbb{R}^{2} \times \mathbb{R}$ be an ensemble of actuated flow snapshots and corresponding control signals, $u_{k}=u\left(x, t_{k}\right), \gamma_{k}=\gamma\left(t_{k}\right)$. As noted above, $u_{0}$, the mean value of the baseline case, is assumed to have been removed from $\left\{u_{k}\right\}$. Define the innovation as

$$
\tilde{u}_{k}=u_{k}-P_{S} u_{k} \text {. }
$$

An optimisation problem on the Hilbert space $\mathbb{H}$ can be defined as finding

$$
\psi^{\star}=\arg \min _{\psi \in \mathbb{H}} E\left\{\left\|\tilde{u}_{k}-\gamma_{k} \psi\right\|^{2}\right\} .
$$

The element $\psi^{*} \in \mathbb{W}$ will be called the actuation mode. Since the squared norm of the velocity represents the energy contained in the flow, among all augmented
POD expansions in the form

$$
u(x, t) \approx u_{0}(x)+\sum_{i=1}^{N} a_{i}(t) \phi_{i}(x)+\gamma(t) \psi(x)
$$

where the input $\gamma$ directly appears as the coefficient of $\psi$, the choice $\psi=\psi^{*}$ is optimal, in the sense that the energy not captured by this expansion achieves its minimum for $\psi=\psi^{*}$. The theorem below summarises the main result of this section, which is the solution to the problem posed in (8).

Theorem 1: Let $J(\psi):=E\left[\left\|\tilde{u}_{k}-\gamma_{k} \psi\right\|^{2}\right]$. Then:

(i) The minimum value of the function $J$ is achieved at $\psi^{*}=E\left[\gamma_{k} \tilde{u}_{k}\right] / E\left[\left(\gamma_{k}\right)^{2}\right]$

(ii) $\psi^{*} \in \mathbb{U}$;

(iii) $\psi^{*} \perp \phi_{i}$ for $i=1, \ldots, N$.

Proof:

(i) Note that

$$
\begin{aligned}
J(\psi) & =E\left[\left\|\tilde{u}_{k}-\gamma_{k} \psi\right\|^{2}\right] \\
& =E\left[\left\|\tilde{u}_{k}\right\|^{2}-2 \gamma_{k}\left\langle\tilde{u}_{k}, \psi\right\rangle+\left(\gamma_{k}\right)^{2}\|\psi\|^{2}\right] .
\end{aligned}
$$

Since $J$ is quadratic in $\psi$ with positive leading coefficient $E\left[\gamma_{k}^{2}\right]$, it has a unique minimum. Computing the first variation of $J$ with respect to $\xi \in \mathbb{W}$

$$
\begin{aligned}
\left.\frac{\mathrm{d}}{\mathrm{d} \delta}\right|_{\delta=0} J(\psi+\delta \xi) \\
=\left.\frac{\mathrm{d}}{\mathrm{d} \delta}\right|_{\delta=0} E\left[\left\|\tilde{u}_{k}\right\|^{2}-2 \gamma_{k}\left\langle\tilde{u}_{k}, \psi+\delta \xi\right\rangle+\gamma_{k}^{2}\|\psi+\delta \xi\|^{2}\right] \\
=\left.E\left[-2 \gamma_{k}\left\langle\tilde{u}_{k}, \xi\right\rangle+\gamma_{k}^{2}\langle\psi+\delta \xi, \xi\rangle+\gamma_{k}^{2}\langle\xi, \psi+\delta \xi\rangle\right]\right|_{\delta=0} \\
=E\left[-2 \gamma_{k}\left\langle\tilde{u}_{k}, \xi\right\rangle+\left(\gamma_{k}\right)^{2}\langle\psi, \xi\rangle+\gamma_{k}^{2}\langle\xi, \psi\rangle\right] \\
=E\left[-2 \gamma_{k}\left\langle\tilde{u}_{k}, \xi\right\rangle+2 \gamma_{k}^{2}\langle\psi, \xi\rangle\right] \\
=E\left[\left\langle-2 \gamma_{k} \tilde{u}_{k}+2\left(\gamma_{k}\right)^{2} \psi, \xi\right\rangle\right] \\
=\left\langle E\left[-2 \gamma_{k} \tilde{u}_{k}+2 \gamma_{k}^{2} \psi\right], \xi\right\rangle .
\end{aligned}
$$

For $\psi$ to be an extremum of $J$ its first variation must vanish $\forall \xi \in \mathbb{U}$. Therefore, $E\left[-2 \gamma_{k} \tilde{u}_{k}+\right.$ $\left.2 \gamma_{k}^{2} \psi^{*}\right]=0$ and thus, by linearity of $E$, $\psi^{*}=E\left[\gamma_{k} \tilde{u}_{k}\right] / E\left[\gamma_{k}^{2}\right]$.

(ii) The fact that $\psi^{*} \in \mathbb{W}$ follows from the fact that $E$ is linear, $\gamma_{k} \tilde{u}_{k} \in \mathbb{H}$ and $E\left[\left(\gamma_{k}\right)^{2}\right] \in \mathbb{R}$.

(iii) To show that $\psi^{*} \perp \phi_{i}$ for $i=1 \ldots N$, first note that $\tilde{u}_{k} \perp S$ for all $k=1 \ldots N$. For any choice of $i, k$

$$
\begin{aligned}
\left\langle\tilde{u}_{k}, \phi_{i}\right\rangle & =\left\langle u-P_{S} u, \phi_{i}\right\rangle=\left\langle u-\sum_{j=1}^{n}\left\langle u, \phi_{j}\right\rangle \phi_{j}, \phi_{i}\right\rangle \\
& =\left\langle u, \phi_{i}\right\rangle-\sum_{j=1}^{n}\left\langle u, \phi_{j}\right\rangle\left\langle\phi_{j}, \phi_{i}\right\rangle=0 .
\end{aligned}
$$


Then, for any $i$, using linearity of $E$ and the inner product, it follows that

$$
\left\langle\psi^{*}, \phi_{i}\right\rangle=\left\langle\frac{E\left[\gamma_{k} \tilde{q}_{k}\right]}{E\left[\gamma_{k}^{2}\right]}, \phi_{i}\right\rangle=\frac{\left.E\left[\gamma_{k} \mid \tilde{q}_{k}, \phi_{i}\right)\right]}{E\left[\gamma_{k}^{2}\right]}=\frac{E[0]}{E\left[\gamma_{k}^{2}\right]}=0
$$

that is, $\psi^{*} \perp S$.

Remark 1: In the above derivations, it is implicity assumed that the subspace $S=\operatorname{span}\left\{\phi_{i}\right\}_{i=1}^{N}$ captures a significant portion of the baseline energy, so that the innovation $\tilde{u}_{k}$ can be attributed almost completely to the effect of the input.

Remark 2: One might notice similarities between the cost function in (8) and the cost function used to compute POD modes in $\S 3$. While in both cases the goal is to minimise the energy neglected by the expansion, the major difference of the proposed method lies in the fact that in (12) the system input $\gamma$ appears directly as a coefficient of the actuation mode $\psi$. This differs from other approaches found in the literature that rely on obtaining the actuation modes from a classical POD expansion on the innovations. For those cases, the modal coefficients have no direct relationship to the system input $\gamma$, and additional methods such as stochastic estimation or weak formulation must be applied to relate $\gamma$ to the modal coefficients.

\subsection{Extension to multiple modes}

It is possible to extend the above procedure to obtain actuation modes for additional inputs or for derivatives of the input. For instance, assume the derivative of the input $\dot{\gamma}(t)$ is available. Let us be given an ensemble of actuated flows $\left\{u_{k}\right\}_{1}^{m} \subset \mathbb{R}^{2}$ and an ensemble of actuation values $\left\{\gamma_{k}\right\}_{1}^{m} \subset \mathbb{R}$ together with their derivatives $\left\{\dot{\gamma}^{k}\right\}_{1}^{m} \subset \mathbb{R}$. As in the previous section we assume that the baseline mean value $u_{0}$ has been removed from $\left\{u_{k}\right\}_{1}^{m}$. The actuation mode $\psi_{1}$ for the input $\gamma$ is first obtained using the procedure outlined in the previous section, that is, $\psi_{1}=\arg \min _{\psi \in H} E$ $\left(\left\|\tilde{u}_{k}-\gamma_{k} \psi\right\|\right)$ Define

$$
\breve{u}:=u_{k}-P_{S_{1}} u_{k},
$$

where $S_{1}:=\operatorname{span}\left\{\phi_{1}, \ldots, \phi_{n}, \psi_{1}\right\}$. Note that, since in general $\left\|\psi_{1}\right\| \neq 1$, one needs to use the projection operator in the form

$$
P_{\psi_{1}} u_{k}=\left\|\psi_{1}\right\|^{-1}\left\langle u_{k}, \psi_{1}\right\rangle \psi_{1} .
$$

The procedure as described in $\S 4$ can be applied with $\breve{u}$ replacing $u$ to obtain an actuation mode for $\dot{\gamma}$ as

$$
\psi_{2}=\arg \min _{\psi \in H} E\left(\left\|\breve{u}_{k}-\dot{\gamma}^{k} \psi\right\|\right)
$$

with $\psi_{2} \perp S_{1}$. This yields an actuated POD expansion of the form

$$
u(x, t) \approx u_{0}(x)+\sum_{i=1}^{N} a_{i}(t) \phi_{i}(x)+\gamma(t) \psi_{1}(x)+\dot{\gamma}(t) \psi_{2}(t) .
$$

Obviously, the procedure can be repeated as needed to incorporate $N_{\text {in }}-1$ derivatives of the input signal to obtain the expansion

$$
u(x, t) \approx u_{0}(x)+\sum_{i=1}^{N} a_{i}(t) \phi_{i}(x)+\sum_{i=1}^{N_{\text {in }}} \gamma^{(i-1)}(t) \psi_{i}(x) .
$$

Remark 3: The computational cost of adding actuation modes to the POD expansion is of interest. Given $M$ snapshots and $N$ baseline modes, computing a single innovation vector $\tilde{\mathrm{u}}_{k}=u_{k}-P_{S} u_{k}$ requires $N$ inner products. Computing $M$ innovations is then of complexity $\mathcal{O}(N M)$. The complexity of computing the actuation mode from (14) is clearly $\mathcal{O}(M)$. Therefore, the complexity of computing one actuation mode is $\mathcal{O}(N M)+\mathcal{O}(M)=\mathcal{O}(N M)$. It is easy to see that this is also the cost of each additional mode to be added following the procedure described above.

\section{Obtaining the reduced-order model}

Consider again the dynamical system (1), and let $V_{1}=u_{0}+\operatorname{span}\left\{\phi_{1}, \ldots, \phi_{n}, \psi_{1}\right\}$. A dynamical system

$$
\dot{r}=X_{V_{1}}(r)
$$

on $V_{1}$ that approximates (1) can be obtained by Galerkin projection as

$$
X_{V_{1}}(r)=P_{V_{1}} X(r) .
$$

The Galerkin projection is optimal in the sense that it minimises $\left\|X_{V_{1}}(r)-X(r)\right\|$. Since $X_{V_{1}}(r)-X(r) \perp V_{1}$, one can write

$$
\left\langle X_{V_{1}}(r)-X(r), \phi_{i}\right\rangle=0, \quad i=1 \ldots N
$$

Substituting $r=u_{0}+a_{j} \phi_{j}+\gamma \psi$ into (11) yields

$$
\begin{array}{r}
\left\langle\dot{a}_{j} \phi_{j}+\dot{\gamma} \psi-X(r), \phi_{k}\right\rangle=0 \\
\dot{a}_{j}\left\langle\phi_{j}, \phi_{k}\right\rangle+\dot{\gamma}\left\langle\psi, \phi_{k}\right\rangle-\left\langle X(r), \phi_{k}\right\rangle=0 \\
\dot{a}_{k}\left\langle\phi_{k}, \phi_{k}\right\rangle-\left\langle X(r), \phi_{k}\right\rangle=0
\end{array}
$$

and thus

$$
\dot{a}_{k}=\left\langle X(r), \phi_{k}\right\rangle .
$$

\subsection{Special case: quadratic dynamics}

Quadratic dynamics

$$
X(q):=C+L(q)+Q(q, q),
$$


where $C$ is a constant term, $L(q)$ a linear term and $Q(q, q)$ a quadratic term in $q$, are of interest since many flow systems such as Burger's and Navier-Stokes equations can be represented in this form. Following the procedure outlined in the previous section, we derive the reduced order system for (13), as this will be useful for the examples presented the following section. Substituting $X$ from (13) into (12) yields

$$
\begin{aligned}
\dot{a}_{k}= & \left\langle X(r), \phi_{k}\right\rangle \\
= & \left\langle C+L\left(u_{0}+a_{i} \phi_{i}+\gamma \psi\right)+Q\left(u_{0}+a_{i} \phi_{i}+\gamma \psi, u_{0}\right.\right. \\
& \left.\left.+a_{j} \phi_{j}+\gamma \psi\right), \phi_{k}\right\rangle \\
= & \left\langle C, \phi_{k}\right\rangle+\left\langle L\left(u_{0}\right), \phi_{k}\right\rangle+\left\langle L\left(\phi_{i}\right), \phi_{k}\right\rangle a_{i}+\left\langle L(\psi), \phi_{k}\right\rangle \gamma \\
& +\left\langle Q\left(u_{0}, u_{0}\right), \phi_{k}\right\rangle+\left\langle Q\left(u_{0}, \phi_{j}\right), \phi_{k}\right\rangle a_{j} \\
& +\left\langle Q\left(u_{0}, \psi\right), \phi_{k}\right\rangle \gamma+\left\langle Q\left(\phi_{i}, u_{0}\right), \phi_{k}\right\rangle a_{i} \\
& +\left\langle Q\left(\phi_{i}, \phi_{j}\right), \phi_{k}\right\rangle a_{i} a_{j}+\left\langle Q\left(\phi_{i}, \psi\right\rangle, \phi_{k}\right\rangle a_{i} \gamma \\
& +\left\langle Q\left(\psi, u_{0}\right), \phi_{k}\right\rangle \gamma+\left\langle Q\left(\psi, \phi_{i}\right), \psi\right\rangle a_{i} \gamma \\
& +\left\langle Q(\psi, \psi), \phi_{k}\right\rangle \gamma^{2} \\
= & F_{k}+G_{1 i k} a_{i}+g_{2 k} \gamma+H_{1 i j k} a_{i} a_{j}+H_{2 i k} a_{i} \gamma+h_{3 k} \gamma^{2},
\end{aligned}
$$

where

$$
\begin{aligned}
F_{k} & =\left\langle C, \phi_{k}\right\rangle+\left\langle L\left(u_{0}\right), \phi_{k}\right\rangle+\left\langle Q\left(u_{0}, u_{0}\right), \phi_{k}\right\rangle \\
G_{1 i k} & =\left\langle L\left(\phi_{i}\right), \phi_{k}\right\rangle+\left\langle Q\left(u_{0}, \phi_{j}\right), \phi_{k}\right\rangle+\left\langle Q\left(\phi_{i}, u_{0}\right), \phi_{k}\right\rangle \\
g_{2 k} & =\left\langle L(\psi), \phi_{k}\right\rangle+\left\langle Q\left(u_{0}, \psi\right), \phi_{k}\right\rangle+\left\langle Q\left(\psi, u_{0}\right), \phi_{k}\right\rangle \\
H_{1 i j k} & =\left\langle Q\left(\phi_{i}, \phi_{j}\right), \phi_{k}\right\rangle \\
H_{3 i k} & =\left\langle Q\left(\phi_{i}, \psi\right), \phi_{k}\right\rangle+\left\langle Q\left(\psi, \phi_{i}\right), \phi_{k}\right\rangle \\
h_{3 k} & =\left\langle Q(\psi, \psi), \phi_{k}\right\rangle .
\end{aligned}
$$

System (14) can be represented in compact form as

$$
\dot{a}=F+G_{1} a+g_{2} \gamma+H_{1}(a, a)+H_{2}(a, \gamma)+h_{3} \gamma^{2} .
$$

In case an input mode $\psi^{2}$ for the derivative of the input $\dot{\gamma}$ is present, one obtains the reduced-order model

$$
\begin{aligned}
\dot{a}= & F+G_{1} a+g_{2} \gamma+g_{3} \dot{\gamma}+H_{1}(a, a)+H_{2}(a, \gamma) \\
& +h_{3} \gamma^{2}+H_{4}(a, \dot{\gamma})+h_{5} \dot{\gamma}^{2}+h_{6} \gamma \dot{\gamma} .
\end{aligned}
$$

The structure of the model for higher-order derivatives of the input is obvious, and thus not given explicitly.

\section{Illustrative example for comparison with another method}

In this section, we consider a simple illustrative example of the 2-dimensional heat equation to present a comparison with a previously proposed input separation method. Although the heat equation belongs to the realm of flow control problems, as it describes conduction, it is considered here to provide the simplest (yet meaningful) comparison with the sub-domain method. A more advanced flow problem will be presented in $\S 7$. The method that we will use for comparison is the sub-domain separation method described in Efe and Ozbay (2003a, b, 2004). The main reason behind this choice lies in the initial motivation of this study, which has been guided by a research effort on cavity flow control (Samimy et al. 2007), where the subdomain separation method was applied in the development of reduced-order modelling for feedback control design. Here, the comparison on the heat equation will be performed in open-loop, as the presence of feedback control tends to reduce the effect of the modelling uncertainties.

Consider the $2 \mathrm{D}$ heat equation

$$
\frac{\partial u}{\partial t}=\mu\left(\frac{\partial^{2} u}{\partial x^{2}}+\frac{\partial^{2} u}{\partial y^{2}}\right)
$$

defined on a square domain $\Omega=[0,1] \times[0,1]$, where $\mu=4$. The initial condition is given as

$$
u(x, y, 0)=16 x(x-1) y(y-1)
$$

while the boundary conditions are specified as in Efe and Ozbay (2003a, b, 2004)

$$
\left.\begin{array}{rl}
u(0,0, t) & =\gamma(t), \quad u(1, y, t)=0, \quad u(x, 1, t)=0, \\
\frac{\partial u}{\partial t}(0, y, t) & =\mu \frac{\partial^{2} u}{\partial y^{2}}(0, y, t), \quad \frac{\partial u}{\partial t}(x, 0, t)=\mu \frac{\partial^{2} u}{\partial x^{2}}(x, 0, t) .
\end{array}\right\}
$$

Note that (17) is in the same form $\dot{u}=X(u)$ used in $\S 5$, with the operator $X=\mu\left(\left(\partial^{2} / \partial x^{2}\right)+\left(\partial^{2} / \partial y^{2}\right)\right)$.

\subsection{Reduced order model for the proposed method}

As described in $\S 4$, we first obtained an actuated POD expansion of the form (9). Using $N=5$ in the expansion (9) is shown to preserve more than $99 \%$ of the energy. Snapshots were taken from MATLAB simulations with the following parameters: spatial grid size was selected as $25 \times 25$ points, the time between adjacent snapshots was chosen as 1 millisecond, the total number of snapshots was taken as 1001, whereas the total simulation time was selected as 1 second. A chirp signal actuation of the form $\gamma(t)=\sin \left(2 \pi 10 t^{3}\right)$ was used to build the input mode $\psi$ from snapshots of the innovation process. The reduced-order model was then obtained, as described in $\S 5$, in the form

$$
\dot{a}_{i}(t)=C+L a_{i}(t)+L_{\text {in }} \gamma(t),
$$


where $\left.C_{i}=\left\langle\mu\left(\partial^{2} / \partial x^{2}\right) u_{0}+\left(\partial^{2} / \partial y^{2}\right) u_{0}\right), \phi_{i}\right\rangle$, and

$$
\begin{aligned}
L_{i j} & =\left\langle\mu\left(\frac{\partial^{2}}{\partial x^{2}} \phi_{i}+\frac{\partial^{2}}{\partial y^{2}} \phi_{i}\right), \phi_{j}\right\rangle, \\
L_{i n, i} & =\left\langle\mu\left(\frac{\partial^{2}}{\partial x^{2}} \psi+\frac{\partial^{2}}{\partial y^{2}} \psi\right), \phi_{i}\right\rangle .
\end{aligned}
$$

\subsection{Reduced order model for sub-domain method}

Another approach proposed in the literature for input separation in model reduction is based on a special treatment of the boundary elements of spatiotemporally discretised PDEs. As proposed herein, the method in question, referred to as sub-domain separation, relies upon the ideas that the finite element representation accurately reconstructs the dynamics of the flow, and second, the process under investigation is composed of coherent modes that are achievable after a suitable decomposition scheme like POD. Here, we give only a brief overview of the methodology, as applied to the 2-dimensional heat equation, and refer the reader interested in the technical details to Efe and Ozbay (2003a, b, 2004).

Consider the 2D heat equation given by (17) and assume the sole excitation is of Dirichlét type at the corner $(x, y)=(0,0)$. Let

$$
u(x, y, t) \approx u_{0}(x, y)+\sum_{i=1}^{N} a_{i}(t) \phi_{i}(x, y)
$$

be the POD approximation of the solution to the given PDE. Naturally, the prescribed solution given above is also valid at the boundaries and this leads to the following observation

$$
\sum_{i=1}^{N} \dot{a}_{i}(t) \phi_{i}(x, y)=\mu \sum_{i=1}^{N} a_{i}(t) \zeta_{i}(x, y)
$$

where $\quad \zeta_{i}(x, y)=\left(\partial^{2} \phi_{i} / \partial x^{2}\right)(x, y)+\left(\partial^{2} \phi_{i} / \partial y^{2}\right)(x, y)$. Taking the inner product of both sides with $\phi_{k}(x, y)$ yields

$$
\dot{a}_{k}=\mu \sum_{i=1}^{N} a_{i}(t)\left\langle\phi_{k}(x, y), \zeta_{i}(x, y)\right\rangle,
$$

where $k=1,2, \ldots, N$. The representation above contains the effect of the boundary excitations implicitly. To remedy this situation, a subdomain $\Omega_{2}$ in which the boundary excitation directly affects the flow is identified, where $\Omega_{2}$ is small enough so that $\zeta_{k}(x, y)$ remains essentially constant for $(x, y) \in \Omega_{2}$. Then, the domain is partitioned as $\Omega=\Omega_{2} \cup \Omega \backslash \Omega_{2}$ and the inner product is computed separately over the two domains as

$$
\begin{aligned}
\left\langle\phi_{k}(x, y), \zeta_{i}(x, y)\right\rangle_{\Omega}= & \left\langle\phi_{k}(x, y), \zeta_{i}(x, y)\right\rangle_{\Omega_{2}} \\
& +\left\langle\phi_{k}(x, y), \zeta_{i}(x, y)\right\rangle_{\Omega \backslash \Omega_{2}} .
\end{aligned}
$$

This basically corresponds to repartitioning the domain by changing the limits of a Riemann integral computed over non-overlapping subdomains embodying the domain of the original integral when they are united. For the 2D heat equation, this approach yields the following model:

$$
\dot{a}_{k}(t)=\mathcal{A} a(t)+\mathcal{B} \gamma(t),
$$

where $a(t)=\left(a_{1}(t), a_{2}(t), \ldots, a_{N}(t)\right)^{\mathrm{T}}$ and the matrices $\mathcal{A}$ and $\mathcal{B}$ are computed as

$$
\begin{aligned}
\mathcal{A}_{k i} & =\mu\left(\left\langle\phi_{k}(x, y), \zeta_{i}(x, y)\right\rangle_{\Omega}-\left\langle\phi_{i}(x, y), \zeta_{k}(x, y)\right\rangle_{\Omega_{2}}\right), \\
\mathcal{B}_{k} & =\left.\mu \zeta_{k}(x, y)\right|_{(x, y)=(0,0)} .
\end{aligned}
$$

\subsection{Simulation results}

Numerical simulations for both reduced-order models were carried out using MATLAB PDE Toolbox, with a uniform spatial grid of $50 \times 50$ points and a time step of 0.01 seconds, for a total of 1 second. The purpose of the simulations is to validate and compare both reduced-order models (19) and (22) in their ability to reconstruct unforced and forces solutions of system (17). Snapshots obtained from the solutions of the unforced system are shown in Figure 2(a), whereas Figure 2(b) shows the trajectories of the system under a ramp actuation in the interval $[-2,2]$. The mean value of the solution, $u_{0}(x, y)$ and the baseline modes are shown in Figure 3(a), and the actuation mode derived using the chirp signal, as described in Section 6.1, is given in Figure 3(b). Note that the excitation used to build the model is different from the one used in the reconstruction of the forced solution. Finally, the POD modes used to construct the sub-domain based reduced-order model are shown in Figure 4. In this case, the data set used to compute the POD basis is given by the snapshots in Figure 2(b).

Let us first present a comparison of the modal coefficients $a_{i}(t)=\left\langle u(x, y, t), \phi_{i}(x, y)\right\rangle$ obtained by projecting directly the snapshots onto each of the two POD bases under consideration (that is, the one derived from baseline and the one derived from actuated solution) to those obtained by solving the corresponding Galerkin system, denoted by $a_{G}(t)$. The result of the comparison is shown in Figure 5. 

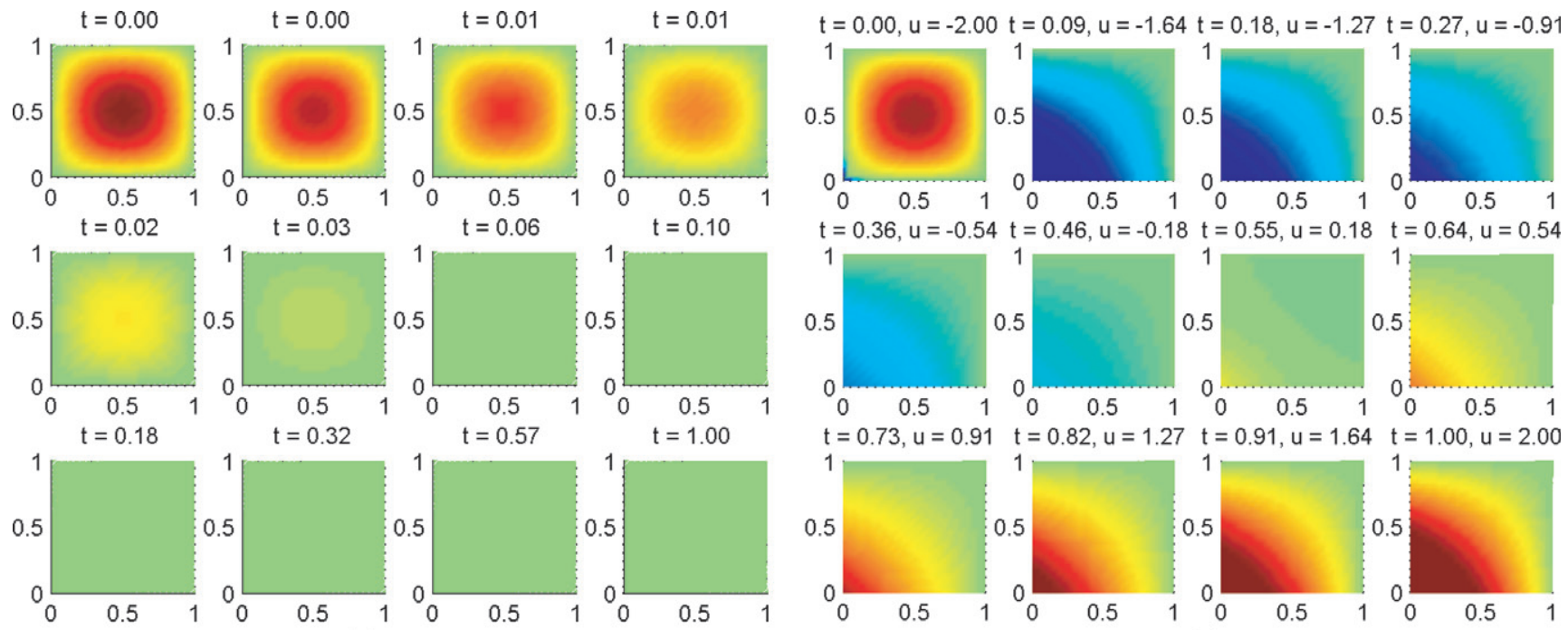

(a)

(b)

Figure 2. Snapshots of solutions of the 2D heat equation: (a) unforced solution; (b) forced solution.

(a)
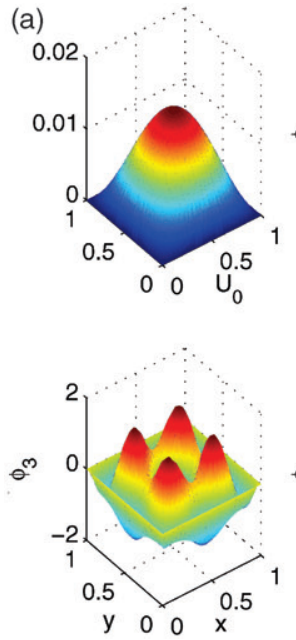
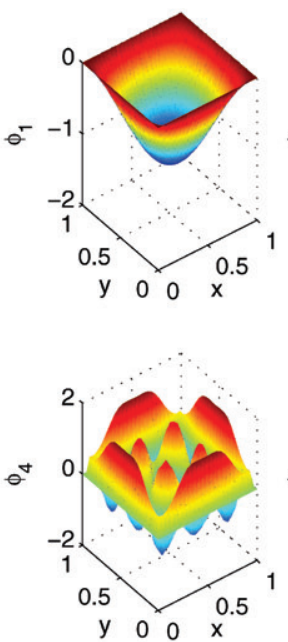
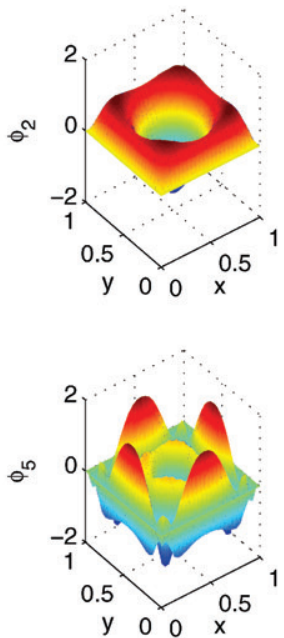

(b)

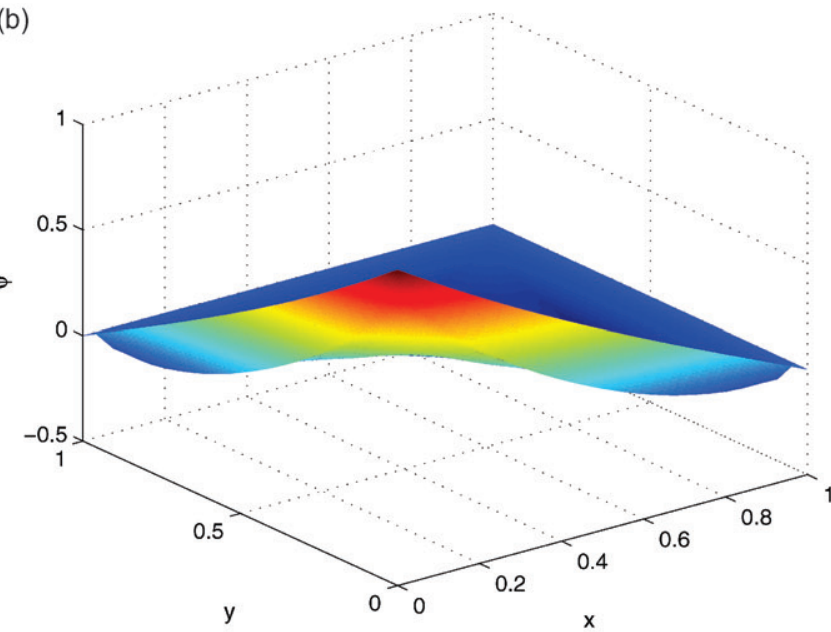

Figure 3. Averaged solution $u_{0}(x, y)$, baseline POD modes $\phi_{i}(x, y)$ and actuation mode $\psi(x, y)$ derived using the proposed method: (a) averaged solution $u_{0}$ and baseline modes $\phi_{i}$; (b) actuation mode $\psi$.
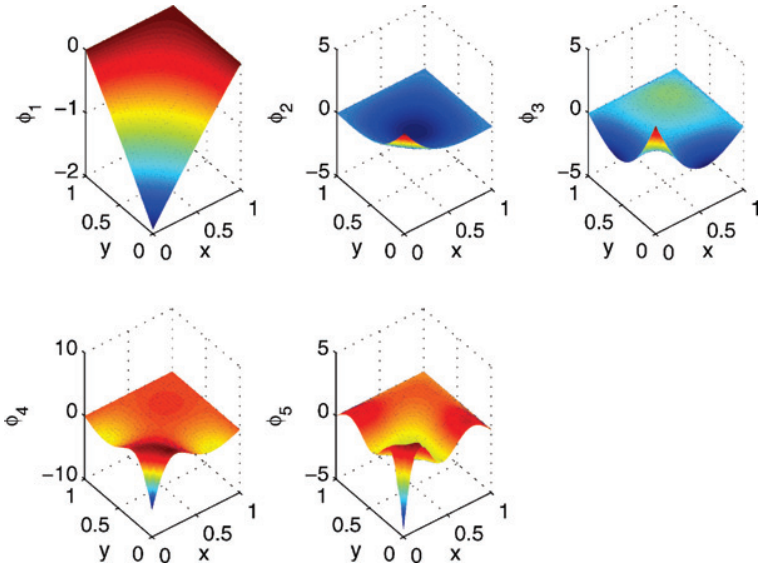

Figure 4. POD modes $\phi_{i}$ obtained using the sub-domain method.
The norm of the error $\left\|a(t)-a_{G}(t)\right\|$ for the reconstruction of the unforced solution is shown in Figure 5(a) and (b) for both methods, whereas the remaining plots in the figure concern the reconstruction of the forced solution. It can be seen that for the proposed method the time histories of the error assume reasonably small values, which suggests good agreement between the full-order and the reducedorder model. For the sub-domain method the error is much larger compared to the proposed method, but the difference for the baseline case is much more pronounced. This can be explained as a result of the fact that a set of snapshots of the actuated solution has been used to build the POD basis, and this 
(a)

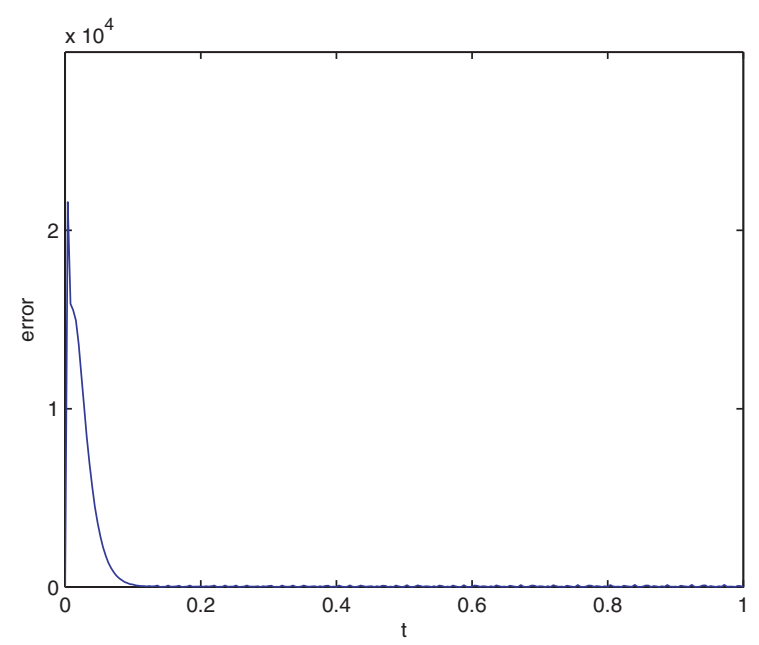

(c)

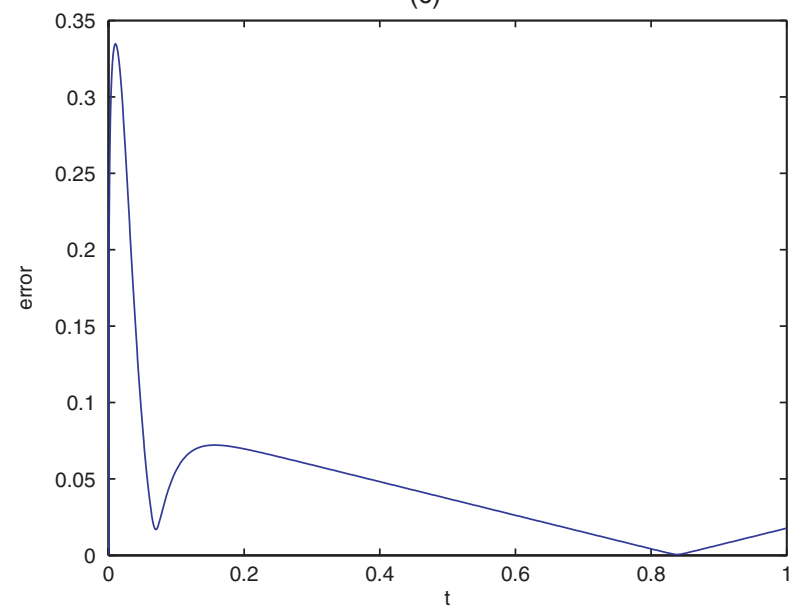

(b)

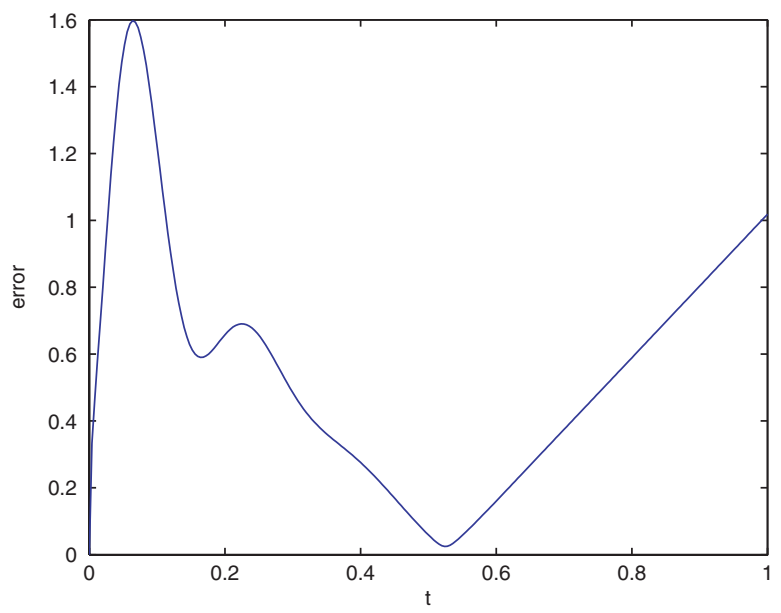

(d)

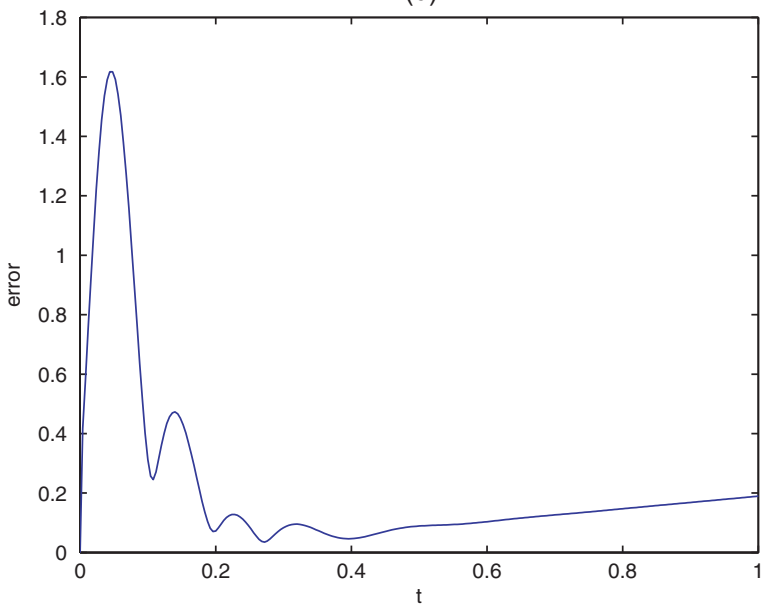

Figure 5. Norm of the error between the actual modal coefficients and those obtained solving the Galerkin model for each method: (a) proposed, unactuated; (b) sub-domain, unactuated; (c) proposed, actuated; (d) sub-domain, actuated.

compromizes the capability of the model to described the baseline case. A symmetric result (not shown here) would be obtained if one uses the baseline snapshots as a data set for the construction of model (22). The reconstruction of the actuated flow is computed by solving (19) and (22) with the given input, and then using the expansions (9) and (20). The results are shown in Figures 6(a) and (b). It can be seen that the proposed method does a much better job in reconstructing the flow during the transient behaviour as compared to the sub-domain method. Also, the reconstruction error reaches a steady state in about 0.03 seconds for the proposed method, whereas it takes about 0.364 seconds for the error obtained from the sub-domain model to decrease to a comparable value.

\section{Example: boundary control of 2D incompressible Navier-Stokes equation}

To illustrate the usefulness and effectiveness of the input separation technique proposed for control design, we consider an example of boundary control of the 2-dimensional Navier-Stokes equation

$$
\frac{\partial U}{\partial t}+(U \cdot \nabla) U=\nabla p+v \Delta U
$$

subject to the incompressibility condition $\nabla \cdot U=0$, where the parameter $v$ is the viscosity, often expressed as $v=R e^{-1}$, where $R e$ is the Reynolds number. Let $U(x, y, t)=(u(x, y, t) v(u, x, t)) \in \mathbb{R}^{2}$ denote the flow variable, where $u$ and $v$ are the components in the longitudinal and latitudinal directions, respectively. 

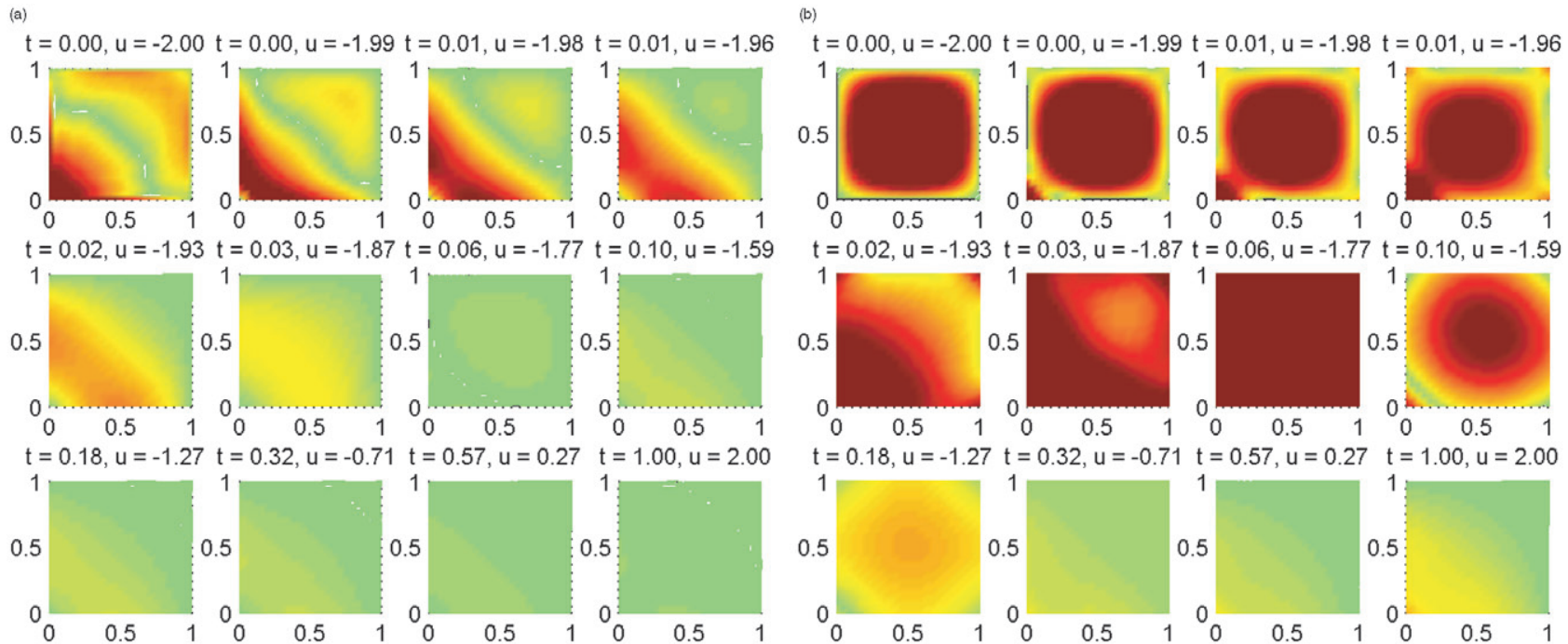

Figure 6. Snapshots of the error between the solution of the heat equation and its reconstruction using reduced-order models: (a) model with proposed input separation method; (b) model with sub-domain separation method.

In the given coordinates, equation (23) reads as

$$
\left.\begin{array}{l}
\frac{\partial u}{\partial t}+\frac{\partial u}{\partial x} u+\frac{\partial u}{\partial y} v=\frac{\partial p}{\partial x}+v\left(\frac{\partial^{2} u}{\partial x^{2}}+\frac{\partial^{2} u}{\partial y^{2}}\right) \\
\frac{\partial v}{\partial t}+\frac{\partial v}{\partial x} u+\frac{\partial v}{\partial y} v=\frac{\partial p}{\partial y}+v\left(\frac{\partial^{2} v}{\partial x^{2}}+\frac{\partial^{2} v}{\partial y^{2}}\right)
\end{array}\right\}
$$

In the considered example, the viscosity is set to $v=0.1$, and the spatial domain is defined as $\Omega=[0,1] \times[0,1]$. The initial conditions $u(x, y, 0)=$ $v(x, y, 0)=0$ and the boundary conditions

$$
\begin{aligned}
& u(x, 0, t)=1 \quad u(x, 1, t)=1 \quad u(0, y, t)=0 \\
& v(x, 0, t)=0 \quad v(x, 1, t)=0 \quad \frac{\partial v}{\partial x}(0, y, t)=0 \\
& u(1, y, t)= \begin{cases}0, & y \in[0,0.42) ; \\
\gamma(t), & y \in[0.42,0.58] ; \quad v(1, y, t)=0 \\
0, & y \in(0.58,1] .\end{cases}
\end{aligned}
$$

are applied, where $\gamma$ is the control input. The solution of the system originating from the given initial condition and subject to the boundary conditions given above, where no $\gamma$ is set to zero, are shown in Figures 7(a) and (b). It can be noted that the trajectories of the unforced system converge to a steady state. Snapshots of the unforced solution were used to derive the baseline POD expansion, where the number of baseline modes selected as $N=3$ can be shown to preserve about $99 \%$ of the energy. The baseline POD modes are shown in Figures 8(a) and (b). The actuation mode $\psi$ was obtained using snapshots of the solution of the forced system, obtained using a chirp signal, that is, an oscillatory excitation of increasing frequency. The solution of (24) under the given forcing is shown in Figure 9, while the actuation mode computed from these snapshots is given in Figure 10. It was observed that different kinds of input excitation produced results for the actuation modes which are very similar to the one shown in the figure.

Following the procedure outline in $\S 5$, one obtains the Galerking system for system (24) as

$$
\dot{a}=C+L a+L_{\text {in }} \gamma+Q(a, a)+Q_{\text {in }} \gamma^{2}+Q_{\text {ain }} a \gamma,
$$

where the constant term is given by

$$
C_{i}=\left\langle\left[\begin{array}{l}
-u_{0} \frac{\partial}{\partial x} u_{0}+v\left(\frac{\partial^{2}}{\partial x^{2}} u_{0}+\frac{\partial^{2}}{\partial y^{2}} u_{0}\right)-v_{0} \frac{\partial}{\partial y} u_{0} \\
-u_{0} \frac{\partial}{\partial x} v_{0}+v\left(\frac{\partial^{2}}{\partial x^{2}} v_{0}+\frac{\partial^{2}}{\partial y^{2}} v_{0}\right)-v_{0} \frac{\partial}{\partial y} v_{0}
\end{array}\right], \phi_{i}\right\rangle
$$



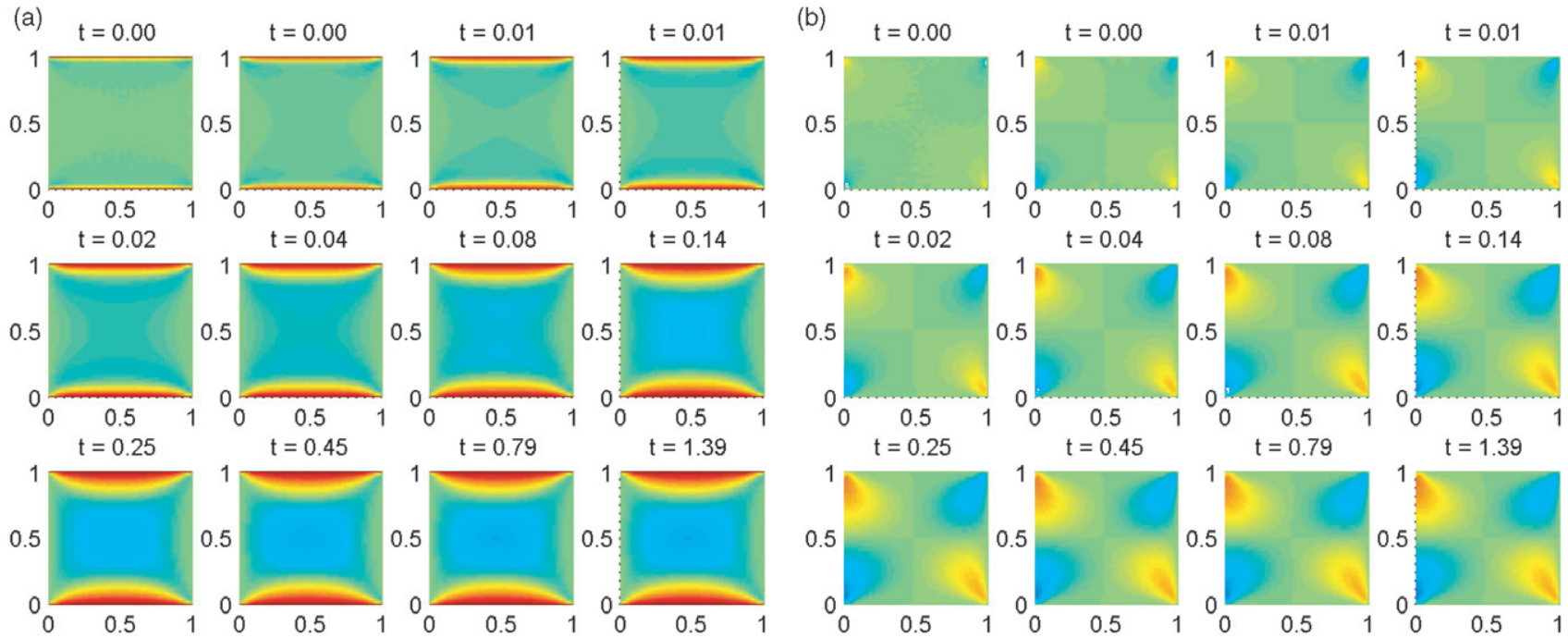

Figure 7. Snapshots of the solution of the unforced Navier-Stokes equation: (a) Snapshots of $u(x ; y ; t)$; (b) Snapshots of $v(x ; y ; t)$.

and the linear and quadratic terms read respectively as

Remark 4: In the above derivations, the pressure term has been neglected, which is standard practice

$$
\begin{aligned}
& \left.L_{i j}=\left\langle\begin{array}{l}
-\left(\phi_{u, j}\right) \frac{\partial}{\partial x} u_{0}+v\left(\frac{\partial^{2}}{\partial x^{2}}\left(\phi_{u, j}\right)+\frac{\partial^{2}}{\partial y^{2}}\left(\phi_{u, j}\right)\right)-\left(\phi_{v, j}\right) \frac{\partial}{\partial y} u_{0} \\
-\left(\phi_{u, j}\right) \frac{\partial}{\partial x} v_{0}+v\left(\frac{\partial^{2}}{\partial x^{2}}\left(\phi_{v, j}\right)+\frac{\partial^{2}}{\partial y^{2}}\left(\phi_{v, j}\right)\right)-\left(\phi_{v, j}\right) \frac{\partial}{\partial y} v_{0}
\end{array}\right], \phi_{i}\right\rangle \\
& L_{\mathrm{in}, i}=\left\langle\left[\begin{array}{c}
-u_{0} \frac{\partial}{\partial x} \psi_{u}-\psi_{v} \frac{\partial}{\partial y} u_{0}+v\left(\frac{\partial^{2}}{\partial x^{2}} \psi_{u}+\frac{\partial^{2}}{\partial y^{2}} \psi_{u}\right)-\psi_{u} \frac{\partial}{\partial x} u_{0}-v_{0} \frac{\partial}{\partial y} \psi_{u} \\
-u_{0} \frac{\partial}{\partial x} \psi_{v}-\psi_{v} \frac{\partial}{\partial y} v_{0}+v\left(\frac{\partial^{2}}{\partial x^{2}} \psi_{v}+\frac{\partial^{2}}{\partial y^{2}} \psi_{v}\right)-\psi_{u} \frac{\partial}{\partial x} v_{0}-v_{0} \frac{\partial}{\partial y} \psi_{v}
\end{array}\right], \phi_{i}\right\rangle \\
& Q_{i j k}=\left\langle\left[\begin{array}{c}
-\left(\phi_{u, k}\right) \frac{\partial}{\partial x}\left(\phi_{u, j}\right)-\left(\phi_{v, k}\right) \frac{\partial}{\partial y}\left(\phi_{u, j}\right) \\
-\left(\phi_{u, k}\right) \frac{\partial}{\partial x}\left(\phi_{v, j}\right)-\left(\phi_{v, k}\right) \frac{\partial}{\partial y}\left(\phi_{v, j}\right)
\end{array}\right], \phi_{i}\right\rangle \\
& Q_{\mathrm{in}, i}=\left\langle\left[\begin{array}{c}
-\psi_{v} \frac{\partial}{\partial y} \psi_{u}-\psi_{u} \frac{\partial}{\partial x} \psi_{u} \\
-\psi_{v} \frac{\partial}{\partial y} \psi_{v}-\psi_{u} \frac{\partial}{\partial x} \psi_{v}
\end{array}\right], \phi_{i}\right\rangle \\
& Q_{\text {ain }, i j}=\left\langle\left[\begin{array}{c}
-\left(\phi_{v, j}\right) \frac{\partial}{\partial y} \psi_{u}-\left(\phi_{u, j}\right) \frac{\partial}{\partial x} \psi_{u}-\psi_{u} \frac{\partial}{\partial x}\left(\phi_{u, j}\right)-\psi_{v} \frac{\partial}{\partial y}\left(\phi_{u, j}\right) \\
-\left(\phi_{v, j}\right) \frac{\partial}{\partial y} \psi_{v}-\left(\phi_{u, j}\right) \frac{\partial}{\partial x} \psi_{v}-\psi_{u} \frac{\partial}{\partial x}\left(\phi_{v, j}\right)-\psi_{v} \frac{\partial}{\partial y}\left(\phi_{v, j}\right)
\end{array}\right], \phi_{i}\right\rangle
\end{aligned}
$$



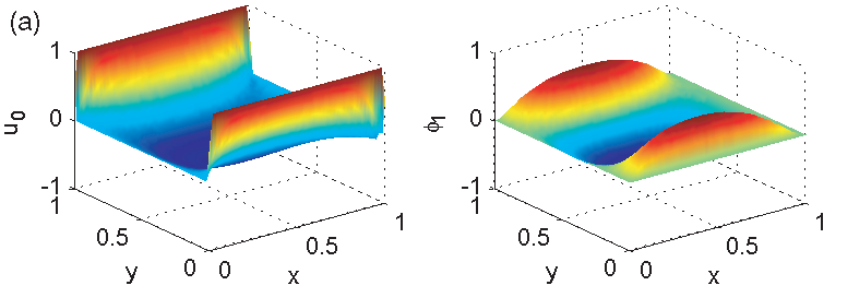

(b)
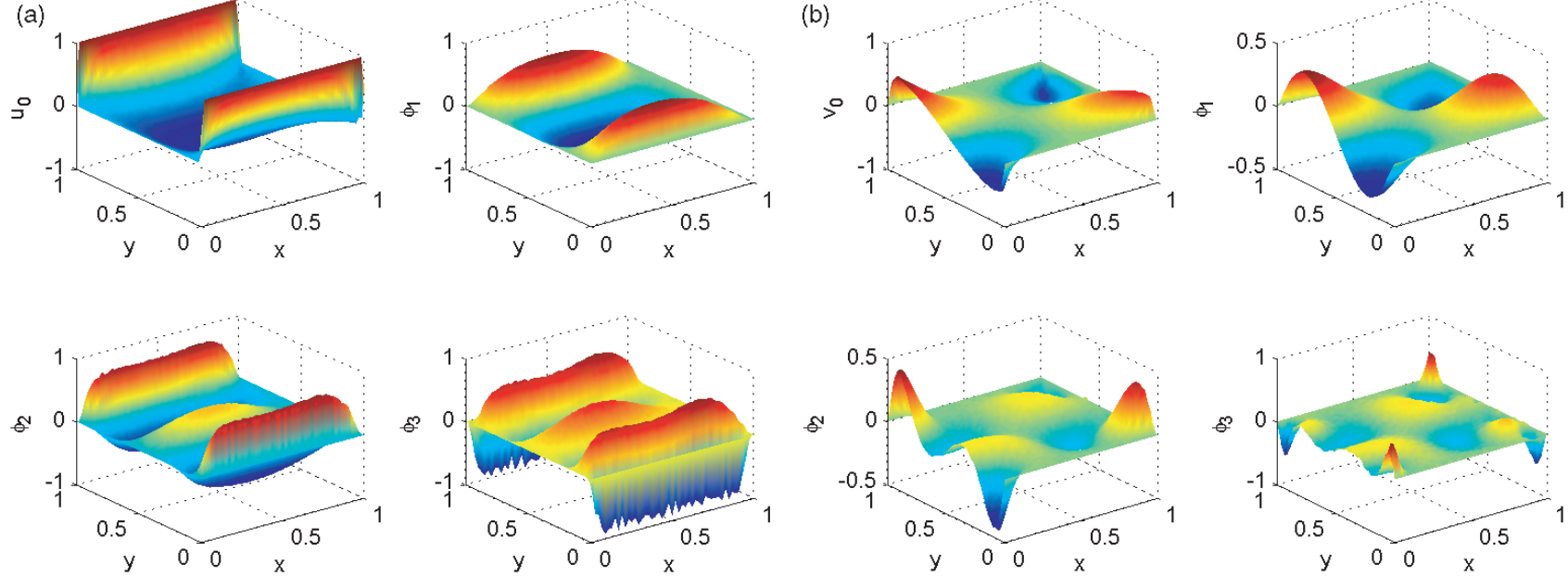

Figure 8. Baseline POD modes for the Navier-Stokes equation: (a) POD modes of $u(x ; y ; t)$ : (b) POD modes of $v(x ; y ; t)$.

(a)

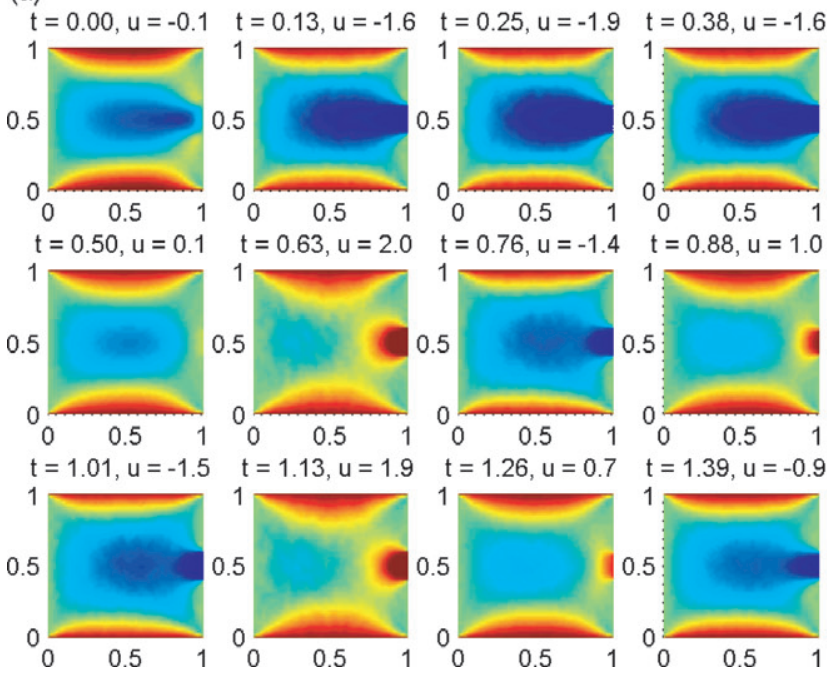

(b)

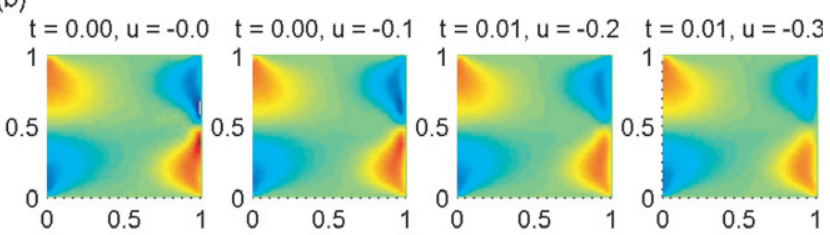
$t=0.02, u=-0.5 \quad t=0.04, u=-0.8 \quad t=0.08, u=-1.3 \quad t=0.14, u=-1.7$

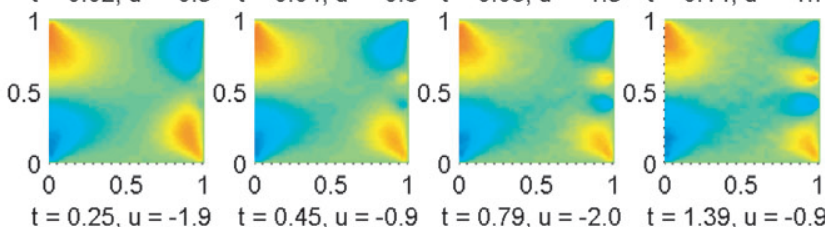
$t=0.25, u=-1.9 \quad t=0.45, u=-0.9 \quad t=0.79, u=-2.0 \quad t=1.39, u=-0.9$

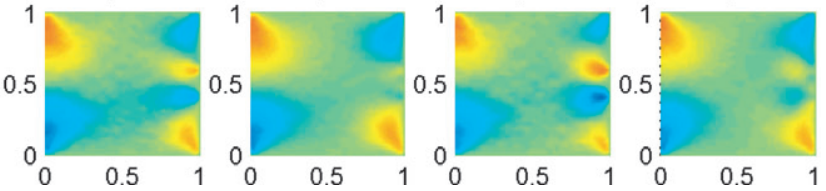

Figure 9. Snapshots of the solution of the actuated Navier-Stokes equation: (a) snapshots of $u(x ; y ; t)$ : (b) snapshots of $v(x ; y ; t)$.
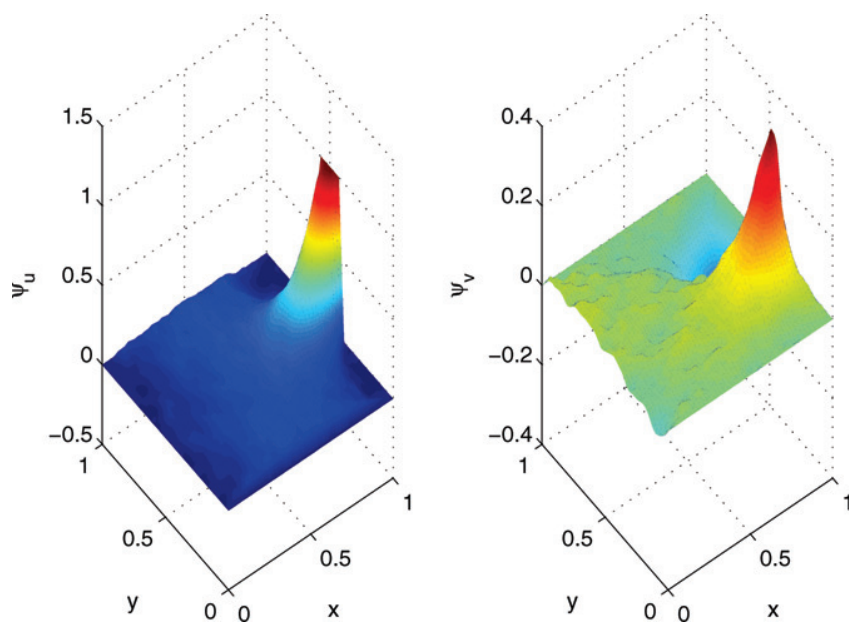

Figure 10. Actuation mode $\psi(x, y)$ for the Navier-Stokes equation. Left plot: $u$-component. Right plot: $v$-component. when obtaining Galerkin models for Navier-Stokes equations. This is either justified theoretically from the boundary conditions or numerically from the CFD simulations. We have applied the latter method by confirming that the effect of the projected pressure terms are indeed negligible. If this is not the case, it is still possible to include the pressure term without changing the model structure (see Noack et al. (2005)).

The control objective selected for this study consists of regulating the trajectories of the systems to a desired 2D flow velocity profile $U_{d}(x, y)=\left(u_{d}(x, y) v_{d}(x, y)\right)$. Since the trajectories generated by the Galerkin system always lie in the set $S=\operatorname{span}\left\{\phi_{i}, \psi\right\}$, the objective is only feasible if $U_{d} \in S$. ${ }^{1}$ Keeping this in mind, we select a desired profile as shown in Figure 11. Note that this 
is a non-equilibrium state of the system in absence of an appropriate control action.

From the desired profile $U_{d}$ one obtains the corresponding vector of desired modal coefficients $a_{d}=\left(a_{d_{i}}\right)_{i=1}^{N}$ as $a_{d_{i}}=\left\langle U_{d}, \phi_{i}\right\rangle$ by projection. Assuming there exists $\gamma_{d}$ such that

$$
C+L a_{d}+L_{\text {in }} \gamma_{d}+Q\left(a_{d}, a_{d}\right)+Q_{\text {in }} \gamma_{d}^{2}+Q_{\text {ain }} a_{d} \gamma_{d}=0
$$

one can define the change of coordinates $\tilde{\mathrm{a}}=a-a_{d}$, $\tilde{\gamma}=\gamma-\gamma_{d}$ which yields the error system

$$
\dot{\tilde{a}}=\tilde{L} \tilde{a}+Q(\tilde{a}, \tilde{a})+\tilde{L}_{\mathrm{in}} \tilde{\gamma}+Q_{\mathrm{in}} \gamma^{2}+Q_{\mathrm{ain}} \tilde{a} \tilde{\gamma}
$$

where

$$
\begin{aligned}
\tilde{L} & =L+Q\left(\cdot, a_{d}\right)+Q\left(a_{d}, \cdot\right)+Q_{\text {ain }} \gamma_{d} \\
\tilde{L}_{\text {in }} & =L_{\text {in }}+2 Q_{\text {in }} \gamma_{d}+Q_{\text {ain }} a_{d} .
\end{aligned}
$$
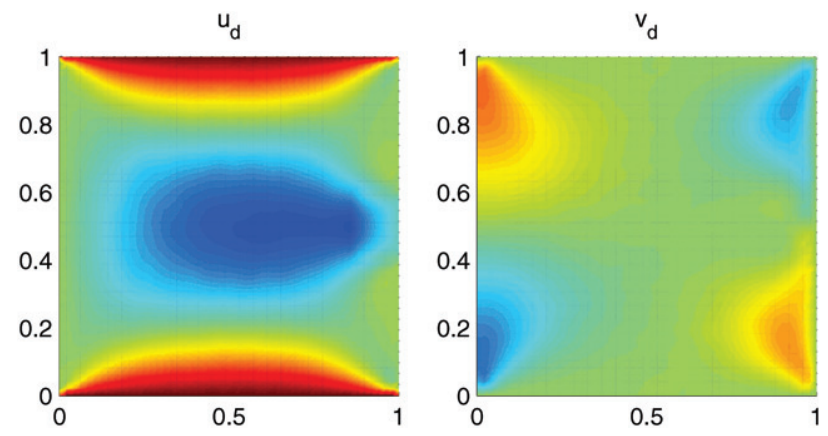

Figure 11. Reference profile for flow velocity.

(a) $\mathrm{t}=0.00, \mathrm{u}=-1.8 \quad \mathrm{t}=0.00, \mathrm{u}=-1.7 \quad \mathrm{t}=0.01, \mathrm{u}=-1.7 \quad \mathrm{t}=0.01, \mathrm{u}=-1.6$
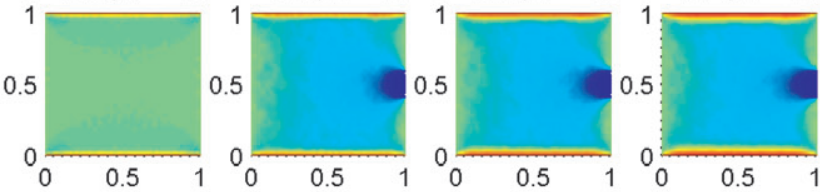

$\mathrm{t}=0.03, \mathrm{u}=-1.5$
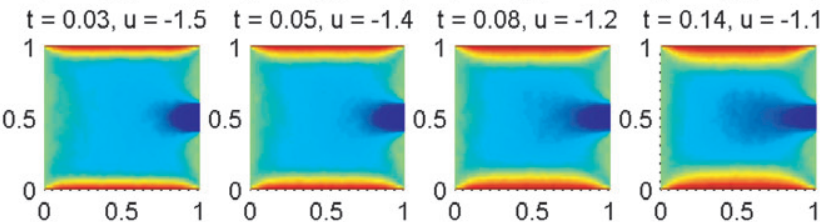

$\mathrm{t}=0.25, \mathrm{u}=-1.0 \quad \mathrm{t}=0.45, \mathrm{u}=-1.0 \quad \mathrm{t}=0.79, \mathrm{u}=-1.0 \quad \mathrm{t}=1.39, \mathrm{u}=-1.0$
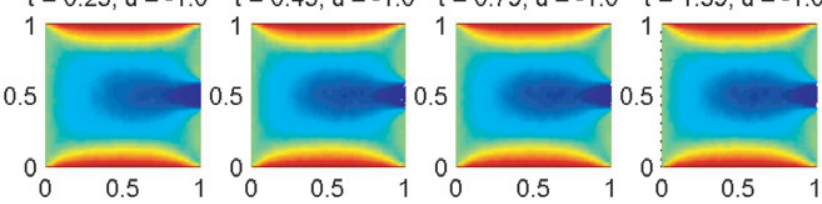

For $\tilde{\gamma}=0$ system (26) has an equilibrium at $\tilde{a}=0$, corresponding to the desired reference state. Therefore, all that is left to be done is to stabilise the equilibrium at the origin. For this study, this has been accomplished by employing a standard LQR control on the linearised system

$$
\dot{\tilde{a}}=\tilde{L} \tilde{a}+\tilde{L}_{i n} \tilde{\gamma}
$$

obtained by minimising the objective function

$$
J(\tilde{\gamma})=\int_{0}^{\infty}\left(\tilde{a}^{T} Q \tilde{a}+\tilde{\gamma}^{T} R \tilde{\gamma}\right) \mathrm{d} t
$$

with $Q=I_{N}$, and $R=0.1$. This yields a feedback controller $\tilde{\gamma}=K \tilde{a}$ with $K=\left[\begin{array}{lll}-0.7972-0.0133 & 0.0914\end{array}\right]$ which places the closed-loop eigenvalues at $[-174.93$ $-8.85-33.46]$, thereby achieving the desired stabilisation. The gains were computed using the lqr command in MATLAB. The controller expressed in the original coordinates is given by $\gamma=\gamma_{d}+K a-K a_{d}$.

Remark 5: Note that control design is based on the Jacobian linearisation of the Galerkin system, and uses feedback from the modal coefficients $a_{i}=\left\langle U, \phi_{i}\right\rangle$. While it is possible to consider more sophisticated control design methodologies, including observerbased design, here a choice has been made to keep the development of the controller as simple as possible and concentrate the attention on the proposed separation method.

Figures 12(a) and (b) show the result of the implementation of the control law in the original Navier-Stokes CFD simulation, while Figure 13 compares the steady-state of the closed-loop system with the desired profile. It can be seen that the

(b)
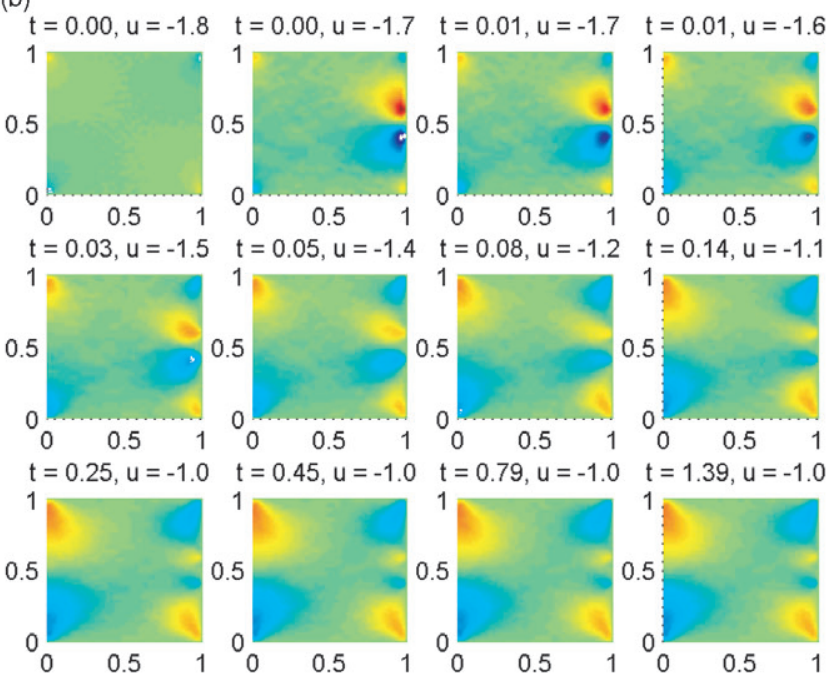

Figure 12. Snapshots of the solution of the controlled Navier-Stokes equation: (a) snapshots of $u(x, y, t)$; (b) snapshots of $v(x, y, t)$. 

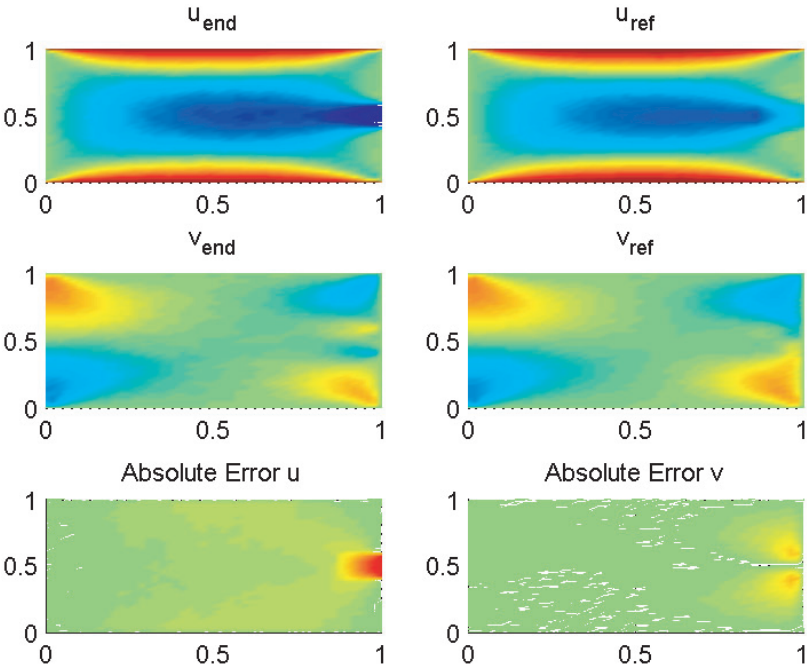

Figure 13. Steady state of controlled flow vs. reference profile.

\subsection{Control design using a two input mode POD based model}

Next, a control design based on a reduced order model obtained from a POD expansion with two actuation modes is investigated. For this purpose, one first needs to compute the actuation mode $\psi_{2}$ for the derivative of the input, $\dot{\gamma}$. This is again accomplished using the actuated snapshots in Figure 9, based on the innovations computed as differences from $\operatorname{span}\left\{\phi_{i}, \psi\right\}$, as described in $\S 4.1$. The result of the computation is shown in Figure 14.

The corresponding Galerkin system is of the form

$$
\begin{aligned}
\dot{a}= & C+L a+L_{\text {in }} \gamma+L_{2 \text { in }} \gamma+Q(a, a)+Q_{\text {in }} \gamma^{2}+Q_{\text {in }} \dot{\gamma}^{2} \\
& +Q_{\text {ain }} a \gamma+Q_{\text {ain }} a \dot{\gamma}+Q_{\text {inin } 2} \gamma \dot{\gamma},
\end{aligned}
$$

where $C, L_{\mathrm{in}}, Q, Q_{\mathrm{in}}, Q_{\text {ain }}$ are given in (25) and

$$
\begin{aligned}
& L_{2 \mathrm{in}, i}=\left.\left\langle\begin{array}{l}
-u_{0} \frac{\partial}{\partial x} \psi_{2 u}-\psi_{2 v} \frac{\partial}{\partial y} u_{0}+v\left(\frac{\partial^{2}}{\partial x^{2}} \psi_{2 u}+\frac{\partial^{2}}{\partial y^{2}} \psi_{2 u}\right)-\psi_{2 u} \frac{\partial}{\partial x} u_{0}-v_{0} \frac{\partial}{\partial y} \psi_{2 u} \\
-u_{0} \frac{\partial}{\partial x} \psi_{2 v}-\psi_{2 v} \frac{\partial}{\partial y} v_{0}+v\left(\frac{\partial^{2}}{\partial x^{2}} \psi_{2 v}+\frac{\partial^{2}}{\partial y^{2}} \psi_{2 v}\right)-\psi_{2 u} \frac{\partial}{\partial x} v_{0}-v_{0} \frac{\partial}{\partial y} \psi_{2 v}
\end{array}\right], \phi_{i}\right\rangle \\
& Q_{2 \mathrm{in}, i}=\langle\left.\left\langle\begin{array}{l}
-\psi_{2 v} \frac{\partial}{\partial y} \psi_{2 u}-\psi_{2 u} \frac{\partial}{\partial x} \psi_{2 u} \\
-\psi_{2 v} \frac{\partial}{\partial y} \psi_{2 v}-\psi_{2 u} \frac{\partial}{\partial x} \psi_{2 v}
\end{array}\right], \phi_{i}\right\rangle \\
& Q_{2 \mathrm{ain}, i j}=\left\langle\begin{array}{l}
\left.\left.-\left(\phi_{v, j}\right) \frac{\partial}{\partial y} \psi_{2 u}-\left(\phi_{u, j}\right) \frac{\partial}{\partial x} \psi_{2 u}-\psi_{2 u} \frac{\partial}{\partial x}\left(\phi_{u, j}\right)-\psi_{2 v} \frac{\partial}{\partial y}\left(\phi_{u, j}\right)\right], \phi_{i}\right\rangle \\
\left.-\left(\phi_{v, j}\right) \frac{\partial}{\partial y} \psi_{2 v}-\left(\phi_{u, j}\right) \frac{\partial}{\partial x} \psi_{2 v}-\psi_{2 u} \frac{\partial}{\partial x}\left(\phi_{v, j}\right)-\psi_{2 v} \frac{\partial}{\partial y}\left(\phi_{v, j}\right)\right]
\end{array}\right] \\
& Q_{\text {inin }, i}=\left\langle\begin{array}{l}
\left.\left.-\left(\psi_{2, u}\right) \frac{\partial}{\partial x}\left(\psi_{1, u}\right)-\left(\psi_{1, u}\right) \frac{\partial}{\partial x}\left(\psi_{2, u}\right)-\left(\psi_{2, v}\right)(x, y) \frac{\partial}{\partial y}\left(\psi_{1, u}\right)-\left(\psi_{1, v}\right) \frac{\partial}{\partial y}\left(\psi_{2, u}\right)\right], \phi_{i}\right\rangle . \\
\left.-\left(\psi_{2, u}\right) \frac{\partial}{\partial x}\left(\psi_{1, v}\right)-\left(\psi_{1, u}\right) \frac{\partial}{\partial x}\left(\psi_{2, v}\right)-\left(\psi_{2, v}\right)(x, y) \frac{\partial}{\partial y}\left(\psi_{1, v}\right)-\left(\psi_{1, v}\right) \frac{\partial}{\partial y}\left(\psi_{2, v}\right)\right]
\end{array}\right.
\end{aligned}
$$

majority of the error is concentrated in the region where the input is applied. This is to be expected since the desired profile selected was a non-equilibrium state under no forcing input. However, in order to render this profile an equilibrium and stabilise it, a feedforward control effort $\gamma_{d}$ must be applied. Apart from the regions where the external excitation is generated, it can be seen that the steadystate is very close to the desired profile and presents a small residual error.
As described in $\S 5$, with a shift of coordinates the above can be transformed into

$$
\begin{aligned}
\dot{\tilde{a}}= & \tilde{L} \tilde{a}+Q(\tilde{a}, \tilde{a})+\tilde{L}_{\mathrm{in}} \tilde{\gamma}+\tilde{L}_{2 \mathrm{in}} \dot{\tilde{\gamma}}+Q_{\mathrm{in}} \tilde{\gamma}^{2} \\
& +Q_{2 \mathrm{in}} \dot{\tilde{\gamma}}^{2}+Q_{\text {ain }} \tilde{a} \tilde{\gamma}++Q_{\text {ain } 2} \tilde{a} \dot{\tilde{\gamma}}+Q_{\text {inin } 2} \tilde{\gamma} \dot{\tilde{\gamma}}
\end{aligned}
$$

and then linearised into the state-space form

$$
\dot{\tilde{a}}=\tilde{L} \tilde{a}+\tilde{L}_{\text {in }} \tilde{\gamma}+\tilde{L}_{2 \text { in }} \dot{\tilde{\gamma}}
$$


The above can be written in the following standard state space form

$$
\left[\begin{array}{c}
\dot{\tilde{a}} \\
\dot{\tilde{\gamma}}
\end{array}\right]=\left[\begin{array}{cc}
\tilde{L} & \tilde{L}_{\text {in }} \\
0 & 0
\end{array}\right]\left[\begin{array}{l}
\tilde{a} \\
\tilde{\gamma}
\end{array}\right]+\left[\begin{array}{c}
\tilde{L}_{2 \text { in }} \\
1
\end{array}\right] \gamma_{2}
$$

where $\gamma_{2}:=\dot{\tilde{\gamma}}$ is the overall input. An LQR design on this system yields a control of the form $\gamma_{2}=K \operatorname{col}\{\tilde{a}, \tilde{\gamma}\}$ where $\quad K=\left[\begin{array}{llll}-0.1237 & 0.0303 & 0.0061 & 1.1650\end{array}\right]$. This control places the system poles at $\left[\begin{array}{llll}-174.7179 & -31.2961 & -6.7433 & -1.1953\end{array}\right]$, thus achieving the desired regulation. Note that the control law can be interpreted as a dynamic controller, since letting $\gamma_{2}=\left[K_{1} k_{2}\right] \operatorname{col}\{\tilde{a}, \tilde{\gamma}\}$ one obtains

$$
\dot{\tilde{\gamma}}=k_{2} \tilde{\gamma}+K_{1} \tilde{a} .
$$
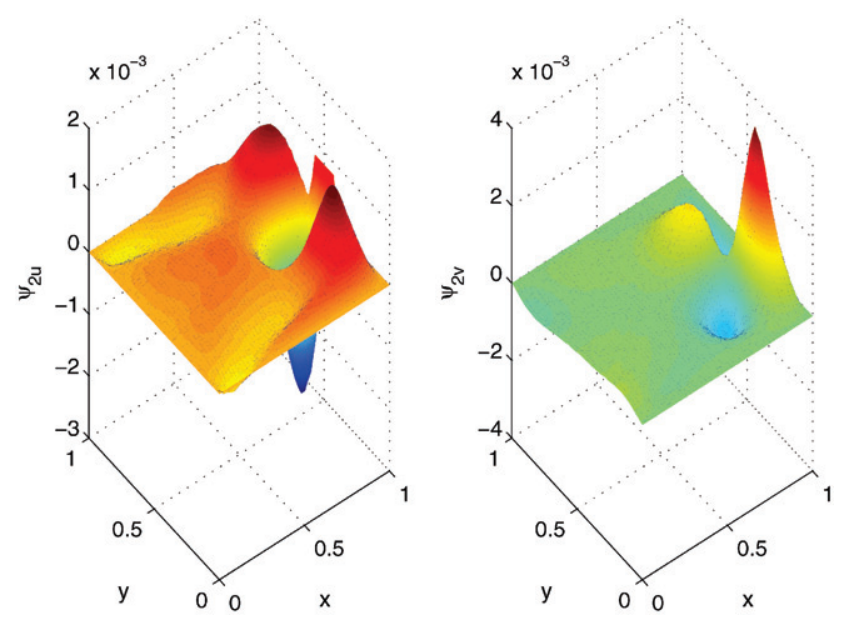

Figure 14. Actuation mode $\psi_{2}(x, y)$.

(a)

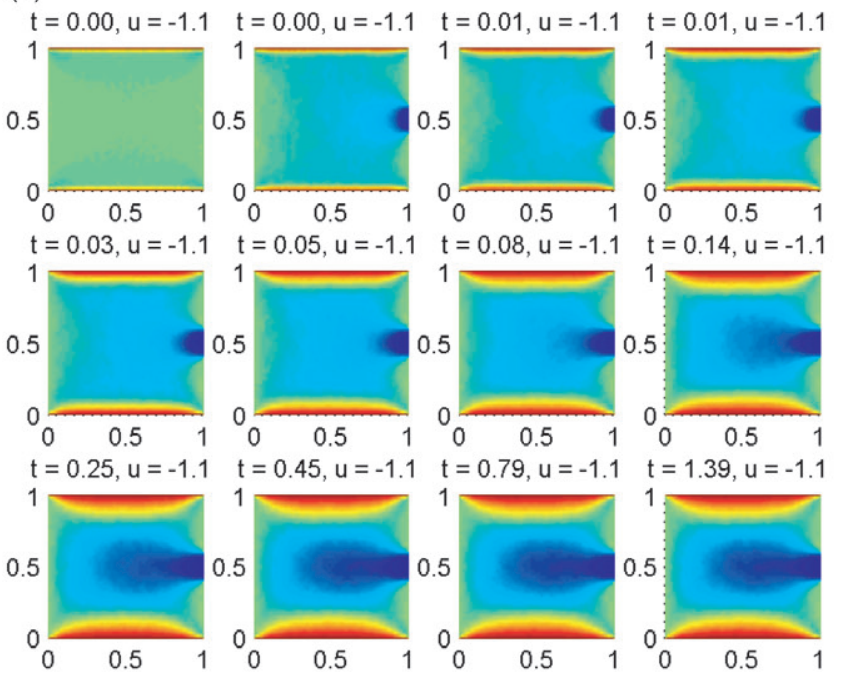

Figures 15(a) and (b) show the result of the implementation of this control in the original Navier-Stokes CFD simulation. Figure 16 compares the final state reached, to the desired profiles. As it was the case for the single actuation mode based control of the previous section, the region of the spatial domain in proximity to the actuation contains the largest portion of the regulation error in steady state. Overall, the results are almost identical to those provided by a reduced-order model with a single actuation mode. As a matter of fact, if one is to compare the results obtained from the two actuation mode based implementation to the one actuation mode based implementation, it is difficult to claim that, for the considered example, the two-modes approach provides a noticeable improvement over the other. This could be expected, since the actuation mode $\psi_{2}$, as seen in Figure 14, is smaller in magnitude compared to the actuation mode for $\gamma$ (see Figure 10). The reason for this behaviour could be traced to the fact that the trend of the input signal may not be as important as its actual value for the dynamics of the system given in this problem. It could also be explained as the property of the input mode $\psi$ to capture and reconstruct most of the effect of the actuation. Consequently, when the new innovations are built as differences from $\operatorname{span}\left\{\phi_{i}, \psi\right\}$, the contribution of $\psi_{2}$ is minimal. Comparing the tracking errors in steady-state for the two models (see Figures 13 and 16), it can be noted that the one-mode approach actually yields a somewhat smaller error in the actuation region, which prompts the question whether the inclusion of an additional model is necessary. At present, this is left to be evaluated, on a case-by-case basis in view of the fact that the two-mode approach obviously requires

(b)

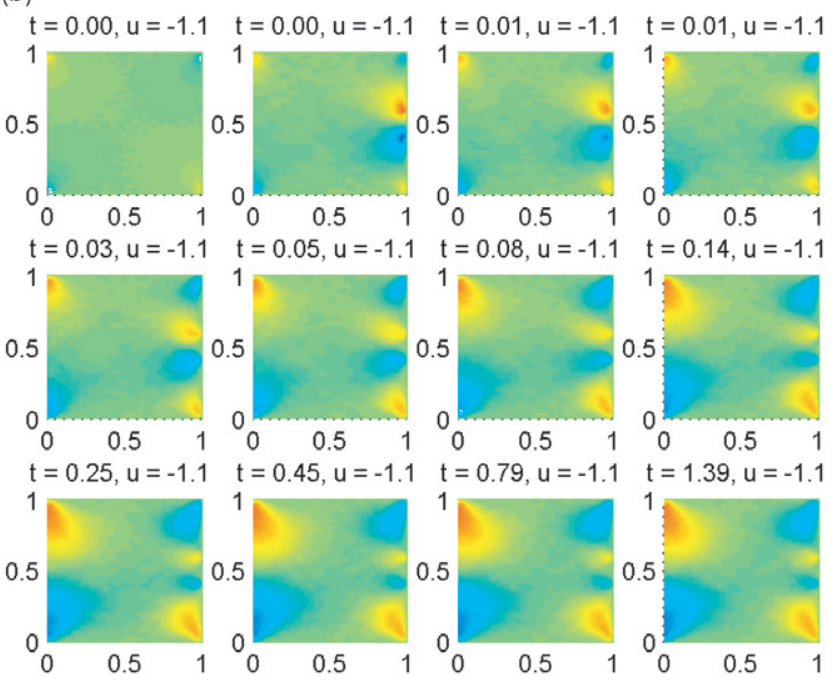

Figure 15. Snapshots of the solution of the controlled Navier-Stokes equation. Model with two actuation modes: (a) snapshots of $u(x, y, t)$; (b) snapshots of $v(x, y, t)$. 

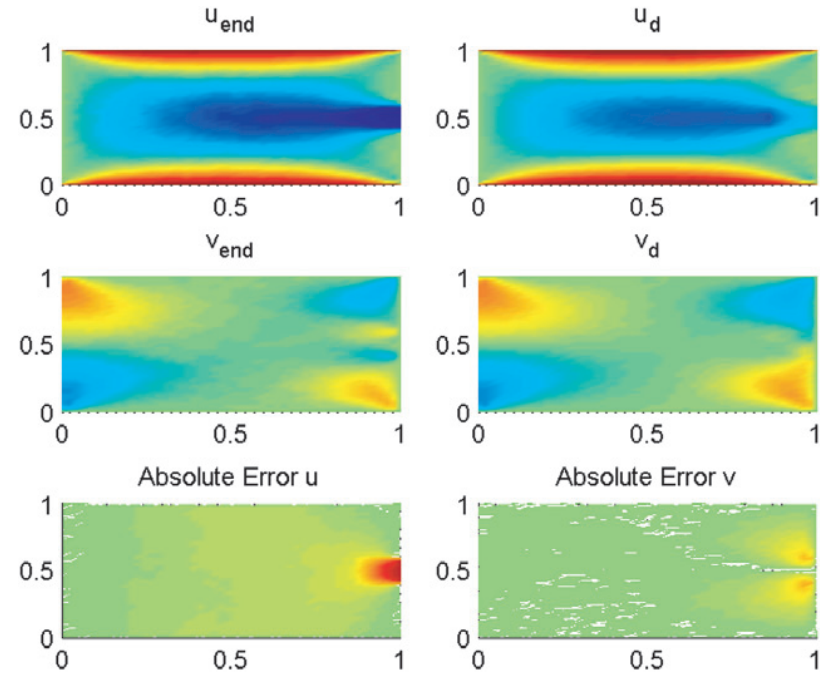

Figure 16. Steady state of controlled flow vs. reference profile for the model with two actuation modes.

a higher computational cost, quantifiable in the computation of a second set of innovations, a second optimisation procedure, and the implementation of a dynamic controller in place of a static one. While, for the given example, the augmentation with an additional mode does not yield an improvement, the situation could be different for more complex dynamics with more intricate interaction with the actuation. Thus, it could still be beneficial to have a systematic methodology for an extension beyond the basic one-mode approach, if needed.

\section{Concluding remarks}

In this paper, an input separation method for the boundary control of flow problems was proposed. First a POD expansion of the unforced baseline case was given. This was then augmented with an optimally chosen actuation mode, which minimises the energy not captured by the augmented set of modes. Then, a reduced-order Galerkin model was derived for this expansion, in which the control input enters the dynamics directly. This reduced-order model was then compared with the one obtained using a subdomain separation method, which is a previously developed technique for input separation. The usefulness of the proposed method was illustrated by a flow control example regarding the boundary control of 2D incompressible Navier-Stokes equations. A controller was designed to achieve a desired spatial profile, using both a model comprising a single actuation mode and one using two actuation modes. It was seen that, in both cases, the reduced-order model-based control laws achieve the desired reference profile in steadystate with small residual error in most of the domain.

The main goal of the study is to obtain a reducedorder model in which the input enters the dynamics explicitly. It is known that the POD modes are optimal, in the sense that the energy neglected by projection onto the subspace spanned by the modes is minimised on average. This notion of optimality, however, does not enforce the boundary conditions per se, and the approximation of the flow field at the boundaries will be as good as it is elsewhere in the spatial domain, regardless of the fact that POD modes are computed using snapshots of the actuated flow of the baseline unforced flow. In the approach presented here, the effect of the input is added a posteriori via an actuation mode, for which the chosen optimality condition requires the input to appear as the modal coefficient. Unlike the standard POD expansion, whose coefficients are computed through projections, the contribution of the actuation term is zero under no forcing, thus the augmented POD expansion reduces exactly to the baseline case. Also, the considered expansion forces all the baseline modes to vanish at any point on the boundary directly influenced by the input where the actuation mode assumes the value one. Hence the augmented expansion at that point will be exactly equal to the value of the input.

It is also worth noting that, although the actuation mode has been obtained from an unconstrained optimisation, one might have reason to impose certain constraints on the problem. For instance, constraints including inner products can be imposed on the actuation mode to make the coefficient computation process similar for both the baseline and actuation modes. We plan on investigating such approaches as part of our future work.

Perhaps the significance and value of the study can be appreciated further if we state that the motivation for this study has arisen from an experimental cavity flow control problem (Samimy et al. 2007). The technique described here was used to develop reduced-order models and feedback controller which achieved comparable or better results than their predecessors in suppressing cavity tone oscillations (Caraballo et al. 2007). For the same problem, methods for further simplification of Galerkin models have been investigated to reveal structural properties to be exploited in more advanced control design (Kasnakoglu and Serrani 2007).

Future directions of this work include extending the proposed method by including actuation modes for multiple inputs, enforcing different constraints on the optimisation problem and applying the methodology to other flow control problems of interest. 


\section{Acknowledgements}

The support of AFOSR/AFRL through the Collaborative Center of Control Science (CCCS) under contract no. F33615-01-2-3154 is gratefully acknowledged. The authors would like to thank Prof. Mo Samimy and colleagues at CCCS for fruitful and stimulating discussions. The first author would also like to thank Darren Engwirda for providing the Navier-Stokes CFD Solver and answering his questions.

\section{Note}

1. If $U_{d} \notin S$, one can attempt to drive the system to $P_{S} U_{d} \in S$

\section{References}

Aamo, O.M., and Krstic, M. (2003), Flow Control by Feedback: Stabilization and Mixing, New York: Springer Verlag.

Aamo, O.M., Krstic, M., and Bewley, T. (2003), "Control of Mixing by Boundary Feedback in 2D Channel Flow," Automatica, 39, 1597-1606.

Antoulas, A.C. (2005), Approximation of Large-Scale Dynamical Systems, Philadelphia, USA: Society for Industrial and Applied Mathematics.

Baramov, L., Tutty, O.R., and Rogers, E. (2004), " $H_{\infty}$ Control of Non-Periodic Two-Dimensional Channel Flow," IEEE Transactions on Control Systems Technology, 12, 111-122.

Bensoussan, A., Prato, D.D., Delfour, M.C., and Mitter, S.K. (1992), Representation and Control of Infinite Dimensional Systems, Boston: Birkhauser.

Burns, J.A., and King, B.B. (1998), "A Reduced Basis Approach to the Design of Low-Order Feedback Controllers for Non-linear Continuous Systems," Journal of Vibration and Control, 4, 297-323.

Cabell, R.H., Kegerise, M.A., Cox, D.E., and Gibbs, G.P. (2002), "Experimental Feedback Control of Flow Induced Cavity Tones," in Proceedings of the 8th AIAA/CEAS Aeroacoustics Conference and Exhibit, Breckenridge, CO, AIAA Paper, pp. 2002-2497.

Camphouse, R.C. (2005), "Boundary Feedback Control Using Proper Orthogonal Decomposition Models," Journal of Guidance, Control, and Dynamics, 28, 931-938.

Caraballo, E., Kasnakoglu, C., Serrani, A., and Samimy, M. (2007), "Control Input Separation Methods for ReducedOrder Model-Based Feedback Flow Control," Accepted by AIAA Journal (in press).

Cattafesta, L., Garg, S., Choudhari, M., and Li, F. (1997), "Active Control of Flow-Induced Cavity Resonance," in Proceedings of the 28th Fluid Dynamics Conference, Snowmass Village, CO, AIAA Paper 97-1804.

Cattafesta, L.N., Shukla, D., Garg, S., and Ross, J.A. (1999), "Development of an Adaptive Weapons-Bay Suppression System" in 5th AIAA/CEAS Aeroacoustics Conference and Exhibit, Vol. 2, Bellevue, WA, USA, AIAA Paper 1999-1901, pp. 676-682.
Curtain, R.F., and Zwart, H.J. (1995), An Introduction to Infinite-Dimensional Linear Systems Theory, New York: Springer Verlag.

Efe, M.O., and Ozbay, H. (2003a), "Integral Action Based Dirichlet Boundary Control of Burgers Equation," in Proceedings of 2003 IEEE Conference on Control Applications, Istanbul, Turkey.

Efe, M.O., and Ozbay, H. (2003b), "Proper Orthogonal Decomposition for Reduced Order Modeling: 2D Heat Flow," in Proceedings of 2003 IEEE Conference on Control Applications, Istanbul, Turkey.

Efe, M.O., and Ozbay, H. (2004), "Low Dimensional Modelling and Dirichlet Boundary Controller Design for Burgers Equation," International Journal of Control, 77, 895-906.

Glauser, M., Higuchi, H., Ausseur, J., and Pinier, J. (2004a), "Feedback Control of Separated Flows," in Proceedings of the 2nd AIAA Flow Control Conference, Portland, OR, USA.

Glauser, M., Young, M., Higuchi, H., and Tinney, C. (2004b), "POD Based Experimental Flow Control on a NACA-4412 Airfoil," in 42nd AIAA Aerospace Sciences Meeting and Exhibit.

Hinze, M., and Kunisch, K. (2004), "Second Order Methods for Boundary Control of the Instationary Navier-Stokes System," Zeitschrift fur Angewandte Mathematik und Mechanik, 84, 171-187.

Holmes, P., Lumley, J., and Berkooz, G. (1996), Turbulence, Coherent Structures, Dynamical System, and Symmetry, Cambridge, UK: Cambridge University Press.

Kasnakoglu, C., and Serrani, A. (2007), "Oscillation Supression in Galerkin Systems Using Center-Manifold and Averaging Techniques," European Journal of Control, $13,1-14$.

Kegerise, M.A., Cattafesta, L.N., and Ha, C. (2002), "Adaptive Identification and Control of Flow-Induced Cavity Oscillations," in 1st AIAA Flow Control Conference, St. Louis, Missouri, AIAA Paper 2002-3158.

King, R., Seibold, M., Lehmann, O., Noack, B.R., Morzynski, M., and Tadmor, G. (2005), Non-linear Flow Control Based on a Low Dimensional Model of Fluid Flow, Berlin Heidelberg: Springer Verlag, pp. 369-386.

Kobayashi, T., and Oya, M. (2003), "Non-linear Boundary Control of Coupled Burgers' Equations," Control and Cybernetics, 32, 245-258.

Kook, H., Mongeau, L., and Franchek, M.A. (2002), "Active Control Of Pressure Fluctuations Due To Flow Over Helmholtz Resonators," Journal of Sound and Vibration, 255, 61-76.

Krstic, M. (1999), "On Global Stabilization of Burgers' Equation by Boundary Control," Systems \& Control Letters, 37, 123-141.

Lawrence, D., Myatt, J., and Camphouse, R. (2005), "On Model Reduction Via Empirical Balanced Truncation," in Proceedings of the 2005 American Control Conference, Portland, Oregon, pp. 3139-3144.

Lehmann, O., Luchtenburg, M., Noack, B., King, R., Morzynski, M., and Tadmor, G. (2005), "Wake Stabilisation using POD Galerkin Models with Interpolated Modes," in Proceedings of the 44th IEEE 
Conference on Decision and Control and 2005 European Control Conference, Seville, Spain, pp. 500-505.

Mongeau, L., Kook, H., and Franchek, M.A. (1998), "Active Control of Flow-Induced Cavity Resonance," in 4th AIAA/CEAS Aeroacoustics Conference, Toulouse, France, AIAA Paper 1998-2349.

Noack, B.R., Papas, P., and Monketwitz, P.A. (2005), "The Need for a Pressure-Term Representation in Empirical Galerkin Models of Incompressible Shear Flows," Journal of Fluid Mechanics, 523, 339-365.

Park, H.M., and Lee, M.W. (2000), "Boundary Control of the Navier-Stokes Equation by Empirical Reduction of Modes," Computer Methods in Applied Mechanics and Engineering, 188, 165-186.

Pillarisetti, A., and Cattafesta, L.N. (2001), "Adaptive Identification of Fluid-Dynamic Systems," in 31st AIAA Fluid Dynamics Conference and Exhibit, Anaheim, CA, AIAA Paper 2001-2978.

Rempfer, D. (2000), “On Low-Dimensional Galerkin Models for Fluid Flow," Theoretical and Computational Fluid Dynamics, 14, 75-88.

Rowley, C.W., Colonius, T., and Murray, R.M. (2000), "POD Based Models of Self-Sustained Oscillations in the Flow Past an Open Cavity," in 6th AIAA/CEAS Aeroacoustics Conference, Lahaina, HI, AIAA Paper 2000-1969.
Rowley, C.W., Colonius, T., and Murray, R.M. (2001), "Dynamical Models for Control of Cavity Oscillations," in 7th AIAA/CEAS Aeroacoustics Conference, Maastricht, Netherlands, AIAA Paper 2001-2126.

Rowley, C.W., Colonius, T., and Murray, R.M. (2004), "Model Reduction for Compressible Flows Using POD and Galerkin Projection," Physica D, 189, 115-129.

Rowley, C., and Williams, D. (2003), "Control of forced and self-sustained oscillations in the flow past a cavity," in Proceedings of the 41st AIAA Aerospace Sciences Meeting, Reno, NV, AIAA paper 2003-0008.

Samimy, M., Debiasi, M., Caraballo, E., Serrani, A., Yuan, X., Little, J., and Myatt, J.H. (2007), "Feedback Control of Subsonic Cavity Flows Using Reduced-Order Models," Journal of Fluid Mechanics, 579, 315-346.

Sirovich, L. (1987), "Turbulence and the Dynamics of Coherent Structures," Quarterly of Applied Math, XLV, 561-590.

Smaoui, N. (2005), "Boundary and Distributed Control of the Viscous Burgers Equation," Journal of Computational and Applied Mathematics, 182, 91-104.

Smith, D., Siegel, S., and McLaughlin, T. (2002), "Modeling of the Wake Behind a Circular Cylinder Undergoing Rotational Oscillation," in 1st AIAA Flow Control Conference, St. Louis, MO, AIAA Paper 2002-3066. 